Physics and operation oriented activities in preparation of the JT-60SA tokamak exploitation

This content has been downloaded from IOPscience. Please scroll down to see the full text.

2017 Nucl. Fusion 57085001

(http://iopscience.iop.org/0029-5515/57/8/085001)

View the table of contents for this issue, or go to the journal homepage for more

Download details:

This content was downloaded by: giruzzi

IP Address: 132.166.84.16

This content was downloaded on 12/07/2017 at 12:07

Please note that terms and conditions apply. 


\title{
Special Topic
}

\section{Physics and operation oriented activities in preparation of the JT-60SA tokamak exploitation}

\author{
G. Giruzzi ${ }^{1}$, M. Yoshida ${ }^{2}$, J.F. Artaud ${ }^{1}$, Ö. Asztalos ${ }^{3}$, E. Barbato ${ }^{4}$, \\ P. Bettini ${ }^{5}$, A. Bierwage ${ }^{6}$, A. Boboc ${ }^{7}$, T. Bolzonella $^{5}$, S. Clement-Lorenzo ${ }^{8}$, \\ S. Coda ${ }^{9}$, N. Cruz ${ }^{10}$, Chr. Day $^{11}$, G. De Tommasi ${ }^{12,13}{ }^{\oplus}$, M. Dibon ${ }^{14}$, \\ D. Douai ${ }^{1}$, D. Dunai ${ }^{15}$, M. Enoeda ${ }^{2}$, D. Farina ${ }^{16}$, L. Figini ${ }^{16}$, M. Fukumoto ${ }^{2}$, \\ K. Galazka ${ }^{17}{ }^{1}$, J. Galdon ${ }^{18}$, J. Garcia ${ }^{1}$, M. Garcia-Muñoz ${ }^{18}$, L. Garzotti ${ }^{7}{ }^{\circ}$, \\ C. Gil ${ }^{1}$, C. Gleason-Gonzalez ${ }^{11}$, T. Goodman ${ }^{9}$, G. Granucci ${ }^{16}$, N. Hayashi ${ }^{2}$, \\ K. Hoshino ${ }^{2}$, S. Ide ${ }^{2}$, R. Imazawa ${ }^{2}$, P. Innocente ${ }^{5}$, A. Isayama ${ }^{2}$, K. Itami ${ }^{2}$, \\ E. Joffrin ${ }^{1}$, Y. Kamada ${ }^{2}$, K. Kamiya ${ }^{2}$, Y. Kawano ${ }^{2}$, H. Kawashima ${ }^{2}$, \\ T. Kobayashi ${ }^{2}$, A. Kojima ${ }^{2}$, H. Kubo ${ }^{2}$, P. Lang ${ }^{14}$, Ph. Lauber ${ }^{14}$, \\ E. de la Luna ${ }^{19}$, P. Maget ${ }^{1}$, G. Marchiori ${ }^{5}$, S. Mastrostefano ${ }^{12,20,}$ \\ G. Matsunaga' ${ }^{2}$, M. Mattei ${ }^{12,21}$, D.C. McDonald ${ }^{22}$, A. Mele ${ }^{12,13}$,Y. Miyata ${ }^{2}$, \\ S. Moriyama ${ }^{2}$, A. Moro ${ }^{16}$, T. Nakano ${ }^{2}$, R. Neu ${ }^{14}$, S. Nowak ${ }^{16}$, F.P. Orsitto ${ }^{4,12}$, \\ G. Pautasso ${ }^{14}$, B. Pégourié ${ }^{1}$, L. Pigatto ${ }^{5}$, A. Pironti ${ }^{12,13}$, P. Platania ${ }^{16}$, \\ G.I. Pokol ${ }^{3}$, D. Ricci' ${ }^{16}$, M. Romanelli ${ }^{7}$, S. Saarelma ${ }^{7}$, S. Sakurai ${ }^{2}$, F. Sartori ${ }^{8}$, \\ H. Sasao ${ }^{2}$, M. Scannapiego ${ }^{11}$, K. Shimizu ${ }^{2}$, K. Shinohara ${ }^{2}$, J. Shiraishi' ${ }^{2}$, \\ S. Soare ${ }^{23}$, C. Sozzi ${ }^{16}$, W. Stępniewski ${ }^{17}$, T. Suzuki ${ }^{2}$, Y. Suzuki ${ }^{24}$, T. Szepesi ${ }^{15}$, \\ M. Takechi' ${ }^{2}$ K. Tanaka ${ }^{24}$, D. Terranova ${ }^{5}$, M. Toma ${ }^{2}$, H. Urano ${ }^{2}$, J. Vega ${ }^{19}$, \\ F. Villone ${ }^{12,20}$, V. Vitale ${ }^{4}$,T. Wakatsuki ${ }^{2}$, M. Wischmeier ${ }^{14}$ and R. Zagórski ${ }^{17}$ \\ 1 CEA, IRFM, F-13108 Saint-Paul-lez-Durance, France \\ 2 National Institutes for Quantum and Radiological Science and Technology, Naka, Ibaraki, 311-0193, \\ Japan \\ ${ }^{3}$ Institute of Nuclear Techniques, Budapest University of Technology and Economics, Budapest, Hungary \\ ${ }^{4}$ EUROfusion/ENEA, Centro Ricerche Frascati, Italy \\ 5 Consorzio RFX, I-35127 Padova, Italy \\ ${ }^{6}$ National Institutes for Quantum and Radiological Science and Technology, Rokkasho, Aomori, 039- \\ 3212, Japan \\ 7 CCFE, Culham Science Centre, Abingdon OX14 3DB, United Kingdom \\ ${ }^{8}$ Fusion for Energy, 08019 Barcelona, Spain \\ 9 EPFL, Swiss Plasma Center, CH-1015 Lausanne, Switzerland \\ 10 Instituto de Plasmas e Fusão Nuclear, Instituto Superior Técnico, Universidade de Lisboa, P-1049-001 \\ Lisboa, Portugal \\ ${ }^{11}$ Institute for Technical Physics, Karlsruhe Institute of Technology, Karlsruhe, Germany \\ 12 Consorzio CREATE, Napoli, Italy \\ 13 Università degli Studi di Napoli Federico II, Napoli, Italy \\ ${ }^{14}$ Max-Planck-Institut für Plasmaphysik, D-85748 Garching, Germany \\ 15 Wigner Research Centre for Physics, Budapest, Hungary \\ 16 Istituto di Fisica del Plasma CNR, 20125 Milano, Italy
}


17 Institute of Plasma Physics and Laser Microfusion, Hery 23, 01-497 Warsaw, Poland

${ }^{18}$ University of Seville, 41012 Seville, Spain

19 Laboratorio Nacional de Fusion, CIEMAT, 28040, Madrid, Spain

${ }^{20}$ Università di Cassino e del Lazio Meridionale, Cassino, Italy

21 Seconda Università degli Studi di Napoli, Aversa, Italy

22 EUROfusion Programme Management Unit, D-85748 Garching, Germany

23 Institute of Cryogenics and Isotopic Technologies-ICIT, 240050, Rm. Valcea, Romania

${ }^{24}$ National Institute for Fusion Science, Toki, Gifu 509-5292, Japan

E-mail: gerardo.giruzzi@cea.fr

Received 16 December 2016, revised 28 March 2017

Accepted for publication 14 June 2017

Published 12 July 2017

\section{Abstract}

The JT-60SA tokamak, being built under the Broader Approach agreement jointly by Europe and Japan, is due to start operation in 2020 and is expected to give substantial contributions to both ITER and DEMO scenario optimisation. A broad set of preparation activities for an efficient start of the experiments on JT-60SA is being carried out, involving elaboration of the Research Plan, advanced modelling in various domains, feasibility and conception studies of diagnostics and other sub-systems in connection with the priorities of the scientific programme, development and validation of operation tools. The logic and coherence of this approach, as well as the most significant results of the main activities undertaken are presented and summarised.

Keywords: tokamak, JT-60SA, modelling, operation, diagnostics

(Some figures may appear in colour only in the online journal)

\section{Introduction}

JT-60SA is a large fully superconducting new tokamak device being built under the Broader Approach Satellite Tokamak Programme jointly by Europe and Japan, and under the Japanese national programme. The JT-60SA tokamak is due to start operation in 2020 [1] and will be at the forefront of the international fusion programme for many years, both before and during the D-T phase of ITER operation. It will support the ITER experimental programme as a satellite machine; at the same time, it will provide key information for the design of DEMO scenarios, in particular for a steady-state, advanced performance design option. Efficient start-up of operation and scientific exploitation of such a large experimental device by an international team is a challenging enterprise, in many aspects similar to what is expected for ITER. A significant amount of resources and experimental time will be required by this initial phase, which can be substantially reduced by adequate preliminary work, in the long years of machine construction. In order to optimize such a start phase, a broad set of preparation activities has been carried out for a few years and is now significantly intensifying. They involve the elaboration of the JT-60SA Research Plan [2], which is regularly updated; advanced modelling in various domains (scenario, MHD and control, fast particles, edge, divertor); feasibility and conception studies of diagnostics and other sub-systems (heating and current drive, matter injection and pumping, tungsten plasma facing components (PFC)) in connection with the priorities of the scientific programme; development and validation of operation tools (data and analysis system, remote participation, magnetic control, wall conditioning). These activities are carried out in a coordinated way by a joint Japan-EU JT-60SA Research Unit, in close interaction with the JT-60SA project for the machine construction [1].

The parameters of JT-60SA, as well as a description of the main plasma scenarios can be found in the JT-60SA Research Plan [2] and are summarized in tables 1 and 2. The most specific characteristics of the machine (size, shaping capability, pulse length, heating and current drive system, diagnostic and control systems) [3] qualify JT-60SA as a tokamak particularly suited for experimental investigation of high beta regimes, fast ion physics, control of high performance scenarios over long pulses [4]. Moreover, by a dedicated experimental programme, JT-60SA will be able to timely address specific ITER risk mitigation issues, such as disruption prevention and mitigation, runaways, ELM avoidance and control, L-H transition, mastering heat loads, test of high-priority diagnostics, real-time control strategies and event handling, etc. JT-60SA can also be used to provide a full-scale test of the ITER data model, analysis and remote participation tools. The activities for preparing the machine exploitation are particularly focused on these scientific goals and results obtained so far are summarised in this article. Modelling results are presented in section 2, including simulations of scenarios, edge and impurities, MHD and energetic particles. Results of critical analysis and conceptual studies of sub-systems with 
Table 1. Nominal parameters of the JT-60SA tokamak.

\begin{tabular}{|c|c|c|c|}
\hline Toroidal field & $2.25 \mathrm{~T}$ & Plasma volume & $132 \mathrm{~m}^{3}$ \\
\hline Plasma current & $5.5 \mathrm{MA}$ & $\begin{array}{l}\mathrm{H} \& \mathrm{CD} \text { power } \\
\text { (total) }\end{array}$ & $41 \mathrm{MW}$ \\
\hline Major/minor radius & $2.96 / 1.18 \mathrm{~m}$ & N-NBI (500 keV) & $10 \mathrm{MW}$ \\
\hline Aspect ratio & 2.5 & P-NBI $(85 \mathrm{keV})$ & $24 \mathrm{MW}$ \\
\hline Elongation & 1.95 & $\begin{array}{l}\text { ECRH }(110,138 \\
\text { GHz) }\end{array}$ & $7 \mathrm{MW}$ \\
\hline Triangularity & 0.53 & Flat-top duration & $100 \mathrm{~s}$ \\
\hline
\end{tabular}

strong impact on the scientific exploitation of the machine are presented in section 3. Operation oriented developments are summarized in section 4. Conclusions and an outlook of the future activities are presented in section 5 .

\section{Modelling}

\subsection{Integrated scenario modelling}

Prediction of the main scenarios is the basis on which all the other activities are built: analysis of the MHD stability, performance of the various sub-systems, operation strategies. In order to develop sound foundations for such predictions, a procedure for validation of models and benchmark of integrated modelling codes has been set up and applied [5], involving the following steps:

- selection and data exchange of reference JT-60U and JET discharges, representing the main scenarios (H-mode, hybrid, advanced).

- selection of various options for transport, pedestal, rotation models and scalings

- predictive simulations of the reference discharges with both Japanese and EU codes.

This procedure had the aim of finding a unified modelling framework that works for the set of reference shots of both machines, which are the most similar in size and characteristics to JT-60SA: this should give the maximum possible confidence for prediction of the JT-60SA scenarios. Moreover, this work has also provided benchmark of various integrated modelling codes, both Japanese and European, with satisfactory results [5], which will allow, in the future, sharing scenario modelling work among various groups. Various transport models have been tested and can be used with comparable accuracy for predictive simulation of most of the reference discharges. However, the main conclusion of this study is that the CDBM heat transport model $[5,6]$ can be safely used for simulation of $\mathrm{H}$-mode, hybrid and advanced scenarios, providing accurate or, in some cases, conservative estimates of the electron and ion temperatures. The validated models and codes have then been used to predict flat-top phases of the main reference JT-60SA scenarios, characterised by the parameters listed in table 2. An example of temperature profiles simulation for Scenario 5-1, but at reduced heating power (24 MW) is shown in figure 1. Both the TOPICS [7] and the CRONOS [8] codes are used, with the CDBM heat transport model and with similar density profiles, magnetic equilibria and pedestal parameters. The two codes compare well, with some differences in the plasma core that can be associated to slight differences in the magnetic equilibria.

Integrated modelling of transient phases (ramp-up, rampdown) is now in progress. In particular, ramp-up strategies with low central solenoid flux consumption (assisted by both NBI and ECCD) have been elaborated by simulations [9] with the TOPICS code, a subject of great importance for access to advanced scenarios in ITER and DEMO, which will be investigated by specific experiments in JT-60SA that has the required long pulse capability. An example of this type of ramp-up scenarios is shown in figure 2. Current drive by NBI and strong heating by ECRH are used during the ramp-up to drive large non-inductive and bootstrap currents in overdrive condition, i.e. with negative Ohmic current (figure $2(a)$ ). The various components of the magnetic flux are shown in figure $2(b)$. Because the threshold $\beta_{\mathrm{N}}>4 l_{\mathrm{i}}\left(\beta_{\mathrm{N}}\right.$ and $l_{\mathrm{i}}$ being normalised beta and internal inductance, respectively) is easily exceeded during this type of ramp-up (figure 2(d)), ideal MHD stability of external kink modes has also been analysed by means of the MARG2D code [10], in the two limiting cases, i.e. with and without a conducting wall, as shown by in figures $2(e)$ and $(f)$. This figure suggests that this type of ramp-up scenarios can be realised without ideal MHD instabilities if the resistive wall modes (RWM) can be stabilised or controlled.

\subsection{Edge and impurity modelling}

The JT-60SA high power, long pulse scenarios will require adequate energy exhaust strategies, based on impurity seeding and control of the radiated power. The machine will start operation with carbon plasma facing components (PFC), then transition to tungsten divertor and first wall is foreseen after achievement of high- $\beta$ plasma scenarios, in order to accompany the ITER experiments (beyond 2029) and to be closer to the expected DEMO scenarios. Therefore, simulations at different approximation levels have been carried out, for both $\mathrm{C}$ and $\mathrm{W}$ environment and exploring various seeding gases. Self-consistent edge-core simulations with simplified (slab) divertor geometry and neutral particles treatment have been performed with the COREDIV code [11, 12], comparing seeding with argon, nitrogen, neon and krypton. An example of this type of simulations for Scenario 3 (high density scenario) is shown in figure 3. It appears that both nitrogen and neon seeding can be used in this scenario to reach radiation fractions up to $80 \%$, with substantial reduction of the power to the divertor plates, but still acceptable $Z_{\text {eff }}$ (effective ion charge number) values. The main difference between the two gases is in the core radiation, which is much stronger for neon. Analogous simulations with $\mathrm{W}$ environment are discussed in section 3.4.

More sophisticated edge-core coupled simulations have been performed combining the integrated modelling code TOPICS and the SOL/divertor code SONIC [13]; impurity transport in the core is computed by the IMPACT code [14]. An example of impurity and temperature profiles obtained in carbon environment with argon seeding for Scenario 5-1 at 
Table 2. Main parameters of the JT-60SA reference scenarios. DN, SN: double null, single null configurations. $I_{\mathrm{p}}$ : plasma current; $B_{\mathrm{T}}$ : toroidal magnetic field; $q_{95}$ : safety factor at $95 \%$ of the poloidal magnetic flux; $P_{\text {add }}$ additional heating power; $P_{\mathrm{NNB}}$ : negative neutral beams power; $P_{\mathrm{PNB}}$ : positive neutral beams power; $P_{\mathrm{EC}}$ : electron cyclotron power; $\bar{n}_{\mathrm{e}}$ : line-averaged electron density; $f_{\mathrm{G}}$ : ratio of the line-averaged electron density to the Greenwald density; $\beta_{\mathrm{N}}$ : normalised plasma beta.

\begin{tabular}{|c|c|c|c|c|c|c|c|c|}
\hline & $\# 1$ & $\# 2$ & \#3 & \#4-1 & \#4-2 & \#5-1 & \#5-2 & $\# 6$ \\
\hline & Inductive & Inductive & High density & ITER-like & $\begin{array}{l}\text { Advanced } \\
\text { inductive }\end{array}$ & $\begin{array}{l}\text { High } \beta \\
\text { full-CD }\end{array}$ & $\begin{array}{l}\text { High } \beta, f_{\mathrm{G}} \\
\text { full-CD }\end{array}$ & $300 \mathrm{~s}$ high $\beta$ \\
\hline Configuration & $\mathrm{DN}$ & SN & $\mathrm{SN}$ & $\mathrm{SN}$ & $\mathrm{SN}$ & $\mathrm{SN}$ & $\mathrm{SN}$ & $\mathrm{SN}$ \\
\hline$I_{\mathrm{p}}(\mathrm{MA})$ & 5.5 & 5.5 & 5.5 & 4.6 & 3.5 & 2.3 & 2.1 & 2.0 \\
\hline$B_{\mathrm{T}}(\mathrm{T})$ & 2.25 & 2.25 & 2.25 & 2.28 & 2.28 & 1.72 & 1.62 & 1.41 \\
\hline$q_{95}$ & 3.2 & 3 & 3 & 3.2 & 4.4 & 5.8 & 6 & 4 \\
\hline$P_{\text {add }}(\mathrm{MW})$ & 41 & 41 & 30 & 34 & 37 & 37 & 30 & 13.2 \\
\hline$P_{\mathrm{NNB}} / P_{\mathrm{PNB}} / P_{\mathrm{EC}}$ & $10 / 24 / 7$ & $10 / 24 / 7$ & $10 / 20 / 0$ & $10 / 24 / 0$ & $10 / 20 / 7$ & $10 / 20 / 7$ & $6 / 17 / 7$ & $3.2 / 6 / 4$ \\
\hline $\bar{n}_{\mathrm{e}}\left(10^{19} \mathrm{~m}^{-3}\right) / f_{\mathrm{G}}$ & $6.3 / 0.5$ & $6.3 / 0.5$ & $10 / 0.8$ & $9.1 / 0.8$ & $6.9 / 0.8$ & $5.0 / 0.85$ & $5.3 / 1.0$ & $2.0 / 0.39$ \\
\hline$\beta_{\mathrm{N}}$ & 3.1 & 3.1 & 2.6 & 2.8 & 3.0 & 4.3 & 4.3 & 3.0 \\
\hline
\end{tabular}

reduced heating power ( $24 \mathrm{MW}$ ) is shown in figure 4. Argon accumulation in the core is obtained (figure $4(a)$ ), owing to neoclassical convection (figure $4(b)$ ) due to bulk density gradient in the core, whereas outward convection due to temperature gradient is dominant in the pedestal. Core radiation due to Ar accumulation causes some temperature decrease in the core (figure $4(c)$ ). Similar simulations, but without seeding, have been performed by means of the JINTRAC code including the EDGE2D SOL/divertor module [15].

\section{3. $M H D$}

The simulated scenarios are then used as a basis for a full set of MHD stability and control simulations: linear and nonlinear behaviour of the edge localised modes (ELM), resistive wall modes (RWM), neoclassical tearing modes (NTM), vertical stability, Alfvénic instabilities driven by fast ions.

2.3.1. Pedestal and ELMs. The reduction of the heat load to the divertor and plasma facing components caused by ELMs is a key issue in ITER. The type I ELM energy losses in JT-60SA are expected to be as large as 10-20\% of the pedestal energy in operational regimes at low collisionality. Therefore, methods to mitigate or suppress large ELMs have been considered in the design of the JT-60SA sub-systems. This includes the use of pellets for ELM pace making and the application of helically resonant magnetic perturbations (RMPs) using the error field control coils (EFCC). Analysis of the 3D plasma response to externally imposed RMPs has shown that the three stacks of coils ( 3 poloidal $\times 6$ toroidal, $30 \mathrm{kAt}$ ) planned for the EFCC system in JT60SA will be sufficient to reach the criteria for ELM suppression based on the Chirikov parameter [16]. In addition to these active ELM control techniques, the investigation of small or no ELM regimes with good performance, such as grassy ELMs or Quiescent H-mode, is also included in the JT-60SA experimental programme, in view of extending the physics basis of such operational regimes toward ITER relevant parameters.

The ELM stability depends on scenario parameters, but in turn it determines the pedestal pressure, which is the fundamental boundary condition for core transport calculations. In order to constrain the pedestal, a series of EPED [17] runs

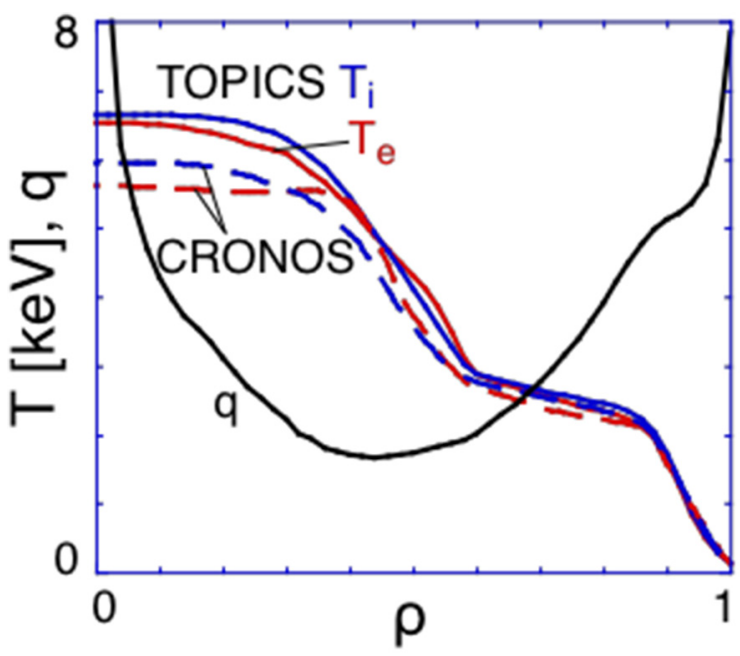

Figure 1. Simulations by the TOPICS (solid lines) and CRONOS (dashed lines) codes for Scenario 5-1, but at reduced heating power (24 MW). Electron (red), ion (blue) temperatures and safety factor (black) profiles versus radial coordinate $\rho$ (square root of normalised toroidal flux) Both codes use the CDBM transport model and similar equilibria and density profiles.

were carried out systematically for the reference JT-60SA scenarios, with assumed pedestal density values. The results of these runs are presented in table 3. High density Scenario (3) and the ITER-like Scenario (4-1) are limited by the high- $n$ ballooning modes, while most others can access the so called 'nose' of the peeling-ballooning mode diagram and become limited by the low- $n$ peeling modes. A specific example of pedestal stability study for Scenario 4-2 (advanced inductive) is shown in figure 5. In the top panel the computed pedestal pressure is plotted as a function of normalised beta $\beta_{\mathrm{N}}$ for various values of the pedestal density. The bottom panel is a plot of the maximum current density in the pedestal region versus the maximum value of the normalised pressure $\alpha$, and it illustrates and explains the behaviour at high beta. At low beta all pedestal pressures, regardless of density, increase with $\beta_{\mathrm{N}}$. However, at high beta the low density is limited by the pure low-n peeling modes, while the high density becomes limited by the intermediate- $n$ peeling-ballooning modes that still benefit from the beta stabilisation effect. 

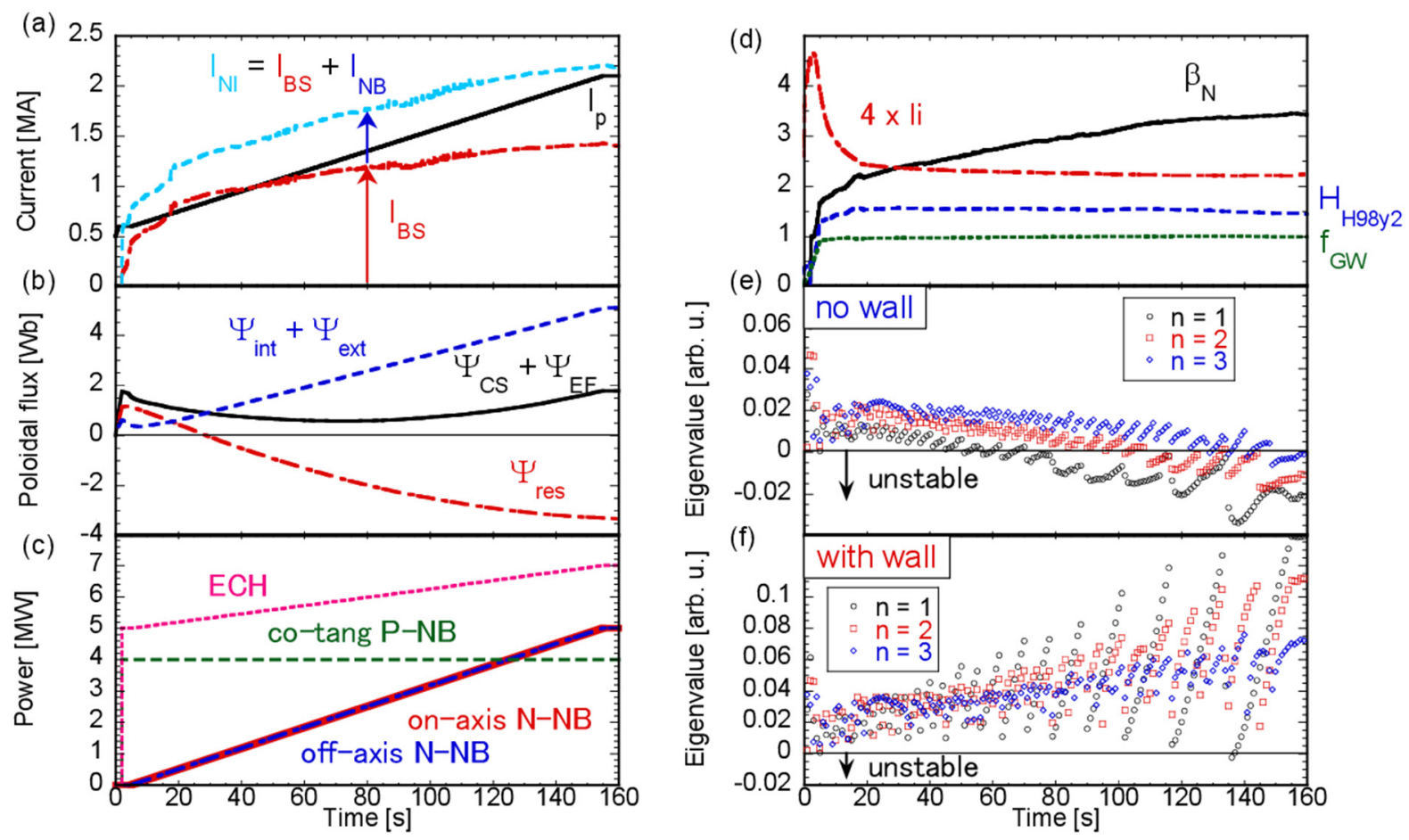

Figure 2. Time evolution of various plasma parameters simulated by the TOPICS $((a)-(d))$ and MARG2D $((e)-(f))$ codes during the rampup phase. (a) Plasma current, bootstrap, NBI-driven and non-inductive current components. (b) Components of the magnetic flux: supplied by the central solenoid (CS) and by the poloidal equilibrium field coils (EF); inductive flux related to magnetic energy stored inside (int) and outside (ext) the plasma boundary; resistive flux (res). (c) Heating power waveforms. (d) $\beta_{\mathrm{N}}, 4 l_{\mathrm{i}}, \mathrm{H}$-factor and Greenwald fraction. (e) Eigen-values of the $n=1-3$ external kink modes without a conducting wall. $(f)$ The same with a perfectly conducting wall.
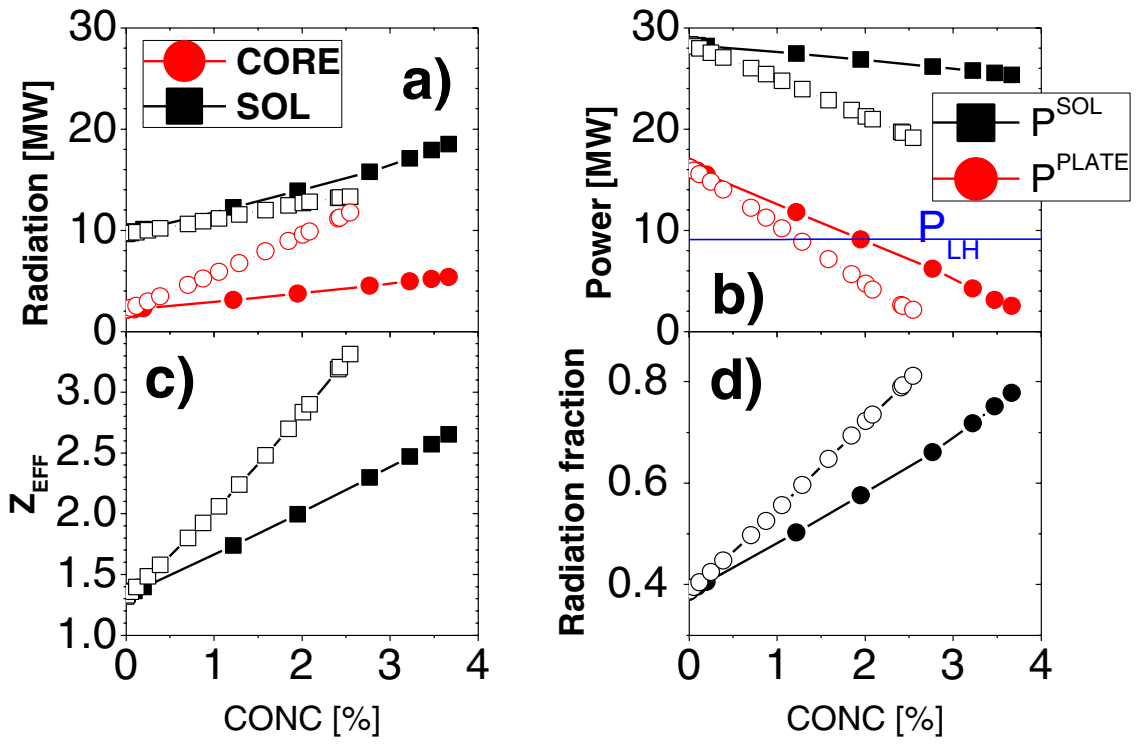

Figure 3. Plasma parameters computed by COREDIV with C-PFC for Scenario 3 versus N (full symbols) and Ne (open symbols) concentrations: $(a)$ core and SOL radiation (red and black, respectively). (b) Power flowing to the divertor plates and to the SOL; in blue: power threshold for the $\mathrm{L}$ to $\mathrm{H}$ mode transition, according to the Martin scaling law. (c) $Z_{\text {eff. }}$ (d) Radiated power fraction.

2.3.2. Resistive wall modes. High-beta scenario stability is a particularly challenging MHD area in which JT-60SA plasmas are expected to give a unique contribution. RWM stability is being studied, including both energetic particles and rotation effects [18], that turn out to be strongly stabilising, when combined. Kinetic effects have also been included in simulations with the 2D MHD code MARS-F/K [19]. A slowing-down energetic particle distribution has been included at present. Substantial RWM damping is found with a combination of drift-kinetic effects with plasma rotation, where the former are represented by the precession drift resonance for trapped particles [20]. An example of how the $n=1$ eigenfunction is modified by the kinetic contribution is given in figure 6 (top panel), while in the bottom panel a 2D map of an $m=2$ harmonic is shown for the fluid non-rotating case. Further developments include the possibility of studying 
anisotropic energetic particle distributions and including additional kinetic resonances for a more detailed description of the RWM damping physics.

Full description of the machine conducting structures, in particular of the stabilising plates, has been implemented in order to study the 3D effects on the stability of the $n=0$ [21] and the $n=1$ [22] RWM ( $n$ being the toroidal mode number), using both CarMa0 [23] and Carma0NL [24] codes. These studies include recovery from perturbations, such as ELMs, minor disruptions, H-to-L transitions etc, which may alter the plasma axisymmetric equilibrium. In particular, the maximum plasma perturbations that can be rejected by any vertical feedback control system have been quantified. Figure 7 shows an example of uncontrolled plasma evolution following a beta drop of 0.54 , corresponding to $92 \%$ of the initial beta, causing the plasma to hit the wall at the inboard side. The eddy currents flowing in the stabilizing plate are shown in the right panel, illustrating the significant effect of holes on the overall pattern.

Active control of the RWM by a specific magnetic coil system will be a key ingredient of these scenarios and is being actively investigated by $3 \mathrm{D}$ electromagnetic computations. The codes CAFE and CARIDDI have been used to characterize the dynamic response of the MHD control system in JT-60SA, in the presence of the 3D conducting structures surrounding the plasma [25]. The ensemble of these studies should allow designing a comprehensive control strategy to cope with RWM and securing the high $\beta_{\mathrm{N}}$ JT-60SA operational space [26]. A proof-of-principle of RWM feedback control has been worked out, for parameters representative of Scenario 5-1, through an eigenvalue study of the closedloop system, which allowed achieving mode stabilization. The result of this first approach, in which only six out of eighteen active coils have been implemented, is shown in figure 8 as a gain scan. It appears that eigenmodes that are strongly unstable in the open loop case can be stabilised if the appropriate closed loop proportional gain is applied. It is worth mentioning that the plasma description underlying these simulations is purely fluid, therefore not including any kinetic damping. Further studies will include all the available actuators and start the preparation of optimized control strategies.

2.3.3. Neoclassical tearing modes. NTMs are expected to be excited in virtually all the JT-60SA scenarios at nominal heating power. Their active control is one of the main functions of the electron cyclotron current drive (ECCD) system, as discussed in section 3.3. The effect of ECCD with the actual antenna configuration is being investigated by solving the Generalized Rutherford Equation, where several terms affecting NTM stability (such as bootstrap, curvature, polarization, non-inductive driven current, heating, wall...) are included. First results are presented and discussed in [26].

\subsection{Energetic particles}

Fast ions driven by NBI and the related Alfvénic instabilities need to be taken into account for the JT-60SA scenario development, with an impact of both their pressure and driven

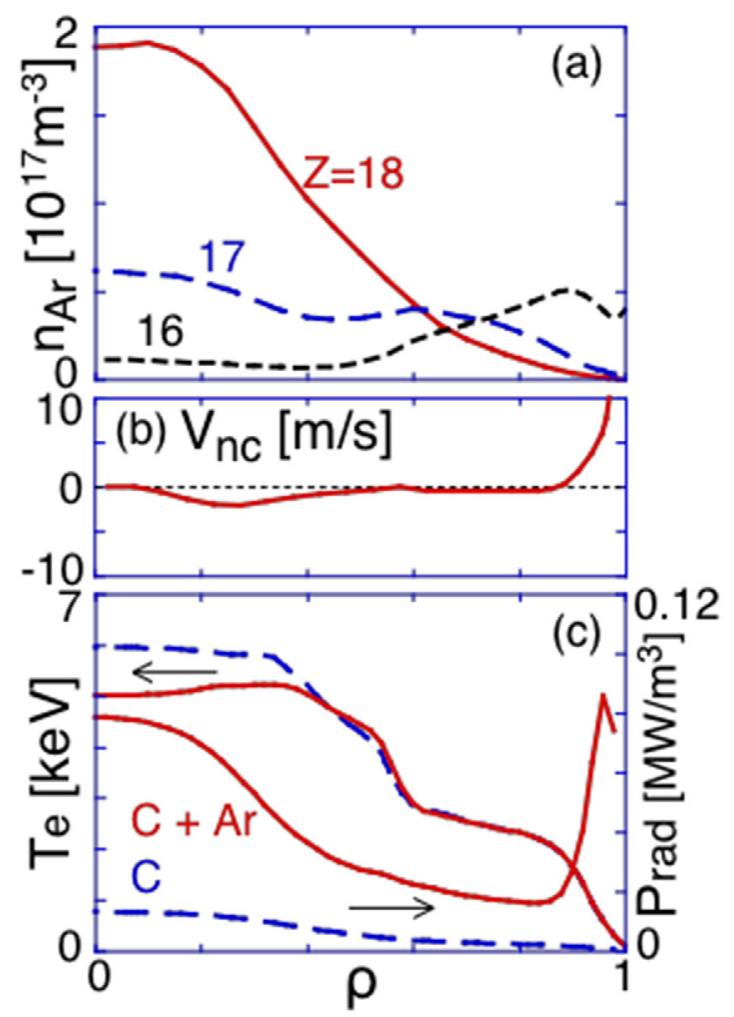

Figure 4. Simulations of Scenario 5-1, but with reduced heating power, with combined integrated modelling code TOPICS, core impurity transport code IMPACT and 2D SOL/divertor code SONIC. Profiles of (a) Ar densities with charge states from $16+$ to $18+;(b)$ neoclassical convective velocities with charge state $18+$; (c) electron temperature and radiated power density with $\mathrm{C}$ only (dashed) or $\mathrm{C}+$ seeded Ar (solid) impurities.

current distributions on the discharge performance. The linear gyrokinetic LIGKA code [27] is used to investigate projected JT-60SA scenarios with respect to energetic particles (EP) driven instabilities. In order to investigate a large number of possible scenarios including sensitivity studies, the fast, local LIGKA version is applied. With this version, ideal and kinetic continua can be calculated. An example of the shear Alfvén mode frequency structure for the high-beta Scenario 5-1 of JT-60SA and toroidal mode number $n=4$ is shown in figure 9 (top). The strong modification (upshift) of the ideal results is due to the large plasma beta characterising this scenario. Further, the radial position, the frequency, the drive/damping and the width of the gap modes for all relevant toroidal mode numbers are automatically determined. The local results are benchmarked and scaled with a few global runs resulting in a very fast and rather accurate linear stability diagram. In the bottom panel of figure 9, toroidal (TAE) and reversed shear (RSAE) Alfvén eigenmodes are shown. Note that the structure of the shear Alfvén continua is sensitive to current and pressure profiles, which in turn can be strongly rearranged by the effect of core MHD modes (e.g. ballooning and double tearing). These can become unstable because of the off-axis radial localisation of the negative-NBI drive (beam energy is 500 $\mathrm{keV})$. Such complex interplay is currently under investigation, in particular using the global non-linear hybrid code MEGA [28]. The goal is to develop more reliable transport models for plasmas with radially localized beam drive. 
Table 3. Pedestal parameters of the reference JT-60SA scenarios, assumed or predicted by the EPED model. From top to bottom: electron density, width (in percentage of normalised magnetic flux), electron temperature and pressure.

\begin{tabular}{lllllllll}
\hline & $\# 1$ & $\# 2$ & $\# 3$ & $\# 4-1$ & $\# 4-2$ & $\# 5-1$ & $\# 5-2$ & $\# 6$ \\
\cline { 2 - 9 } & Inductive & Inductive & $\begin{array}{l}\text { High } \\
\text { density }\end{array}$ & ITER-like & $\begin{array}{l}\text { Advanced } \\
\text { inductive }\end{array}$ & $\begin{array}{l}\text { High } \beta \\
\text { full-CD }\end{array}$ & $\begin{array}{l}\text { High } \beta, \mathrm{f}_{\mathrm{G}} \\
\text { full-CD }\end{array}$ & $\begin{array}{l}300 \mathrm{~s} \\
\text { high } \beta\end{array}$ \\
\hline$n_{\mathrm{e}, \mathrm{ped}}\left(10^{19} \mathrm{~m}^{-3}\right)$ & 5.6 & 5.6 & 9.0 & 8.1 & 6.2 & 4.2 & 4.3 & 1.8 \\
$\Delta\left(\% \Psi_{\mathrm{N}}\right)$ & 3.9 & 4.0 & 3.6 & 3.8 & 4.3 & 4.7 & 4.8 & 4.2 \\
Predicted $T_{\mathrm{e}, \text { ped }}(\mathrm{keV})$ & 2.4 & 3.1 & 1.4 & 1.1 & 1.1 & 1.1 & 1.0 & 1.3 \\
Predicted $p_{\text {ped }}(\mathrm{kPa})$ & 40 & 45 & 36 & 27 & 21 & 12 & 11 & 7 \\
\hline
\end{tabular}
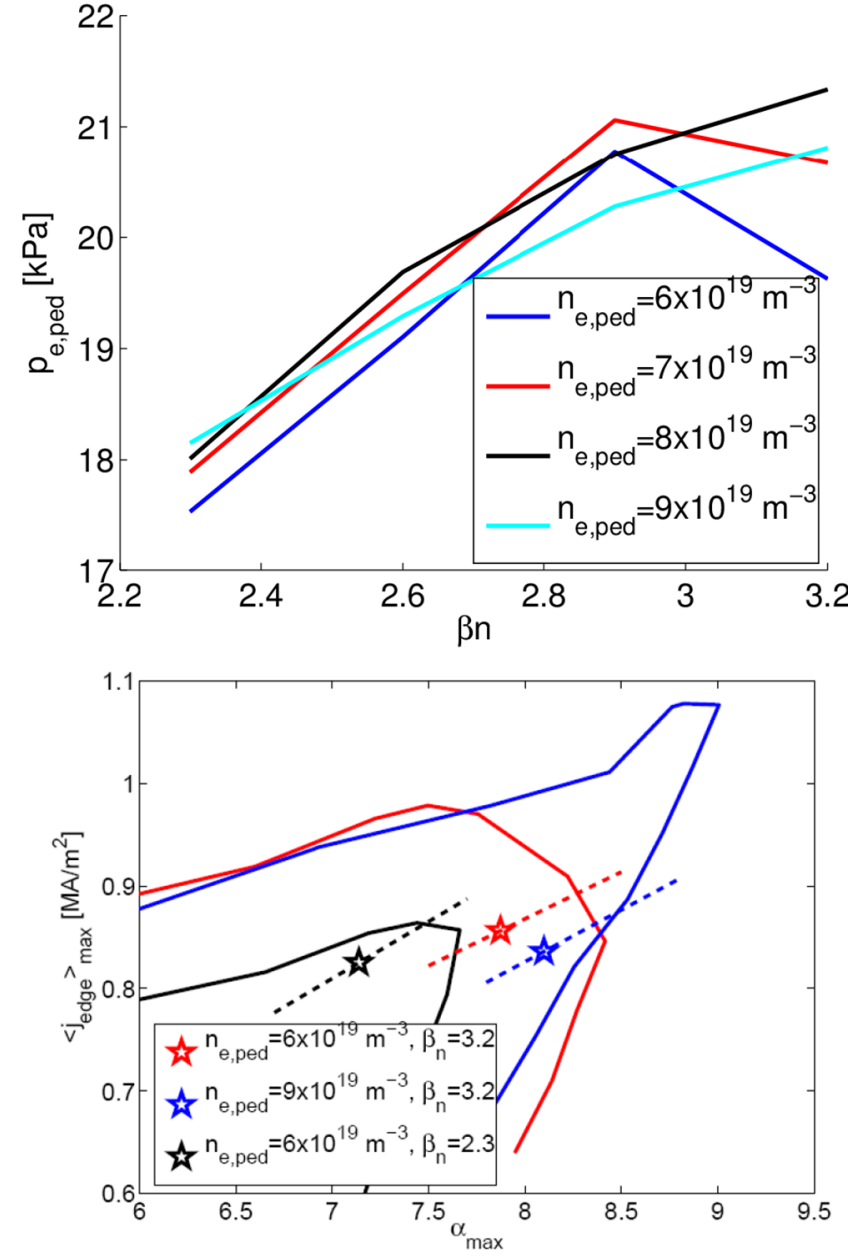

Figure 5. Results of pedestal studies with the EPED model based on parameters of Scenario 4-2 (advanced inductive). Top: pedestal electron pressure versus normalized beta for various pedestal electron densities. Bottom: stability diagrams (limiting the stable and unstable regions) for three working points with different pedestal densities and beta. The dashed lines represent the path that the EPED prediction follows when it crosses the stability boundary.

\section{Sub-systems}

\subsection{Diagnostics}

Feasibility and conceptual studies are being performed to evaluate and qualify the use of various diagnostics (besides the baseline diagnostics that will be available from the first plasma on ([2], App. D]), in connection with the main scientific objectives of the Research Plan or with operation needs. Some relevant examples are presented in this section.
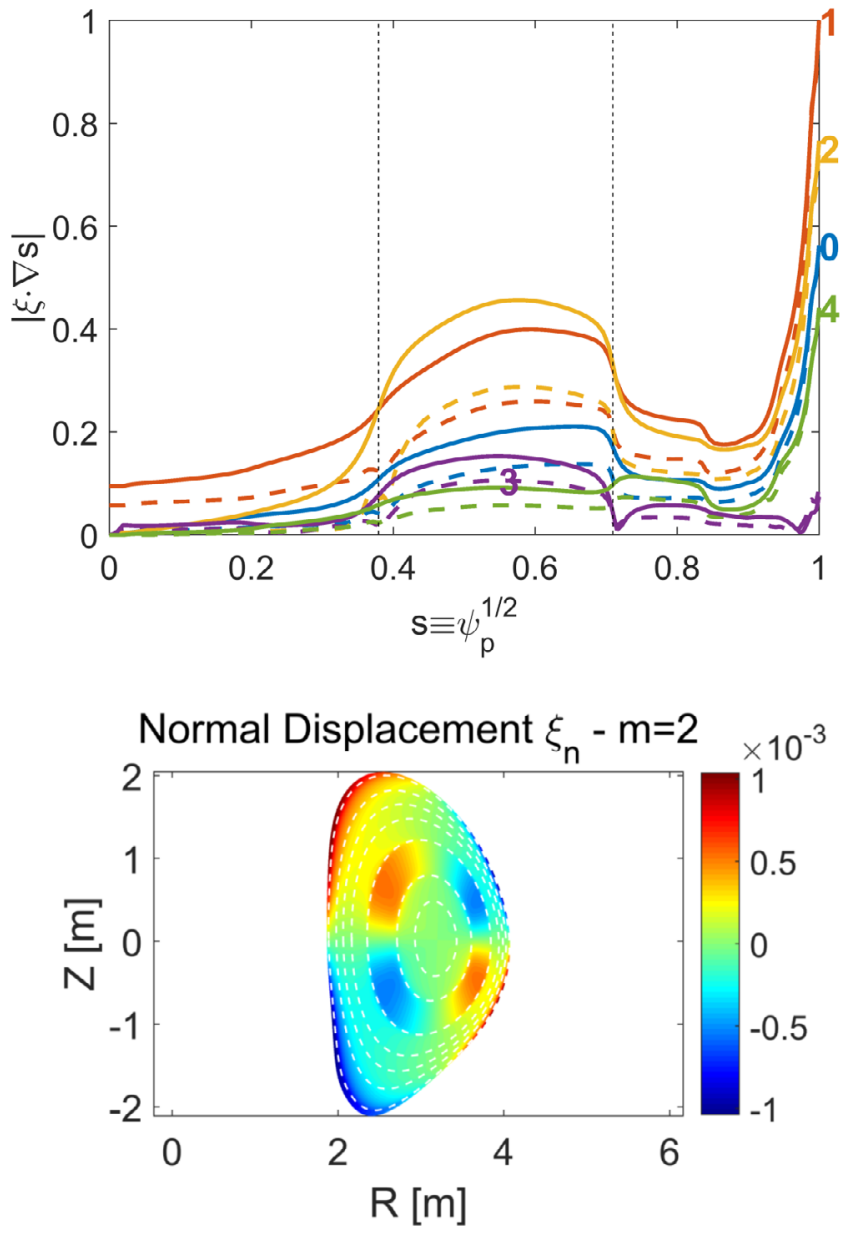

Figure 6. RWM computations with the MARS-F/K code for parameters representative of Scenario 5-1. Top: comparison of RWM eigenfunctions between fluid (solid) and kinetic theory (dashed) including thermal and energetic particles, without rotation. The $q=2$ surfaces are marked with dashed vertical lines. Fourier harmonics of the perturbed radial displacement are given in equalarc coordinate system. Bottom: Normal component of the perturbed plasma displacement, $m=2$ harmonic, calculated by MARS-F in a non-rotating case.

3.1.1. Polarimetry. Diagnosis of the poloidal field profile by polarimetry has been studied in connection with the control of the current profile in advanced high-beta scenarios. A conceptual design of a multi-channel polarimeter driven by realistic 3D-CAD and physics scenarios has shown not only that this system meets the measurement requirements (maximum error of 0.2 degrees in the Faraday rotation angle and operational over the whole plasma discharge cycle) but also that it 

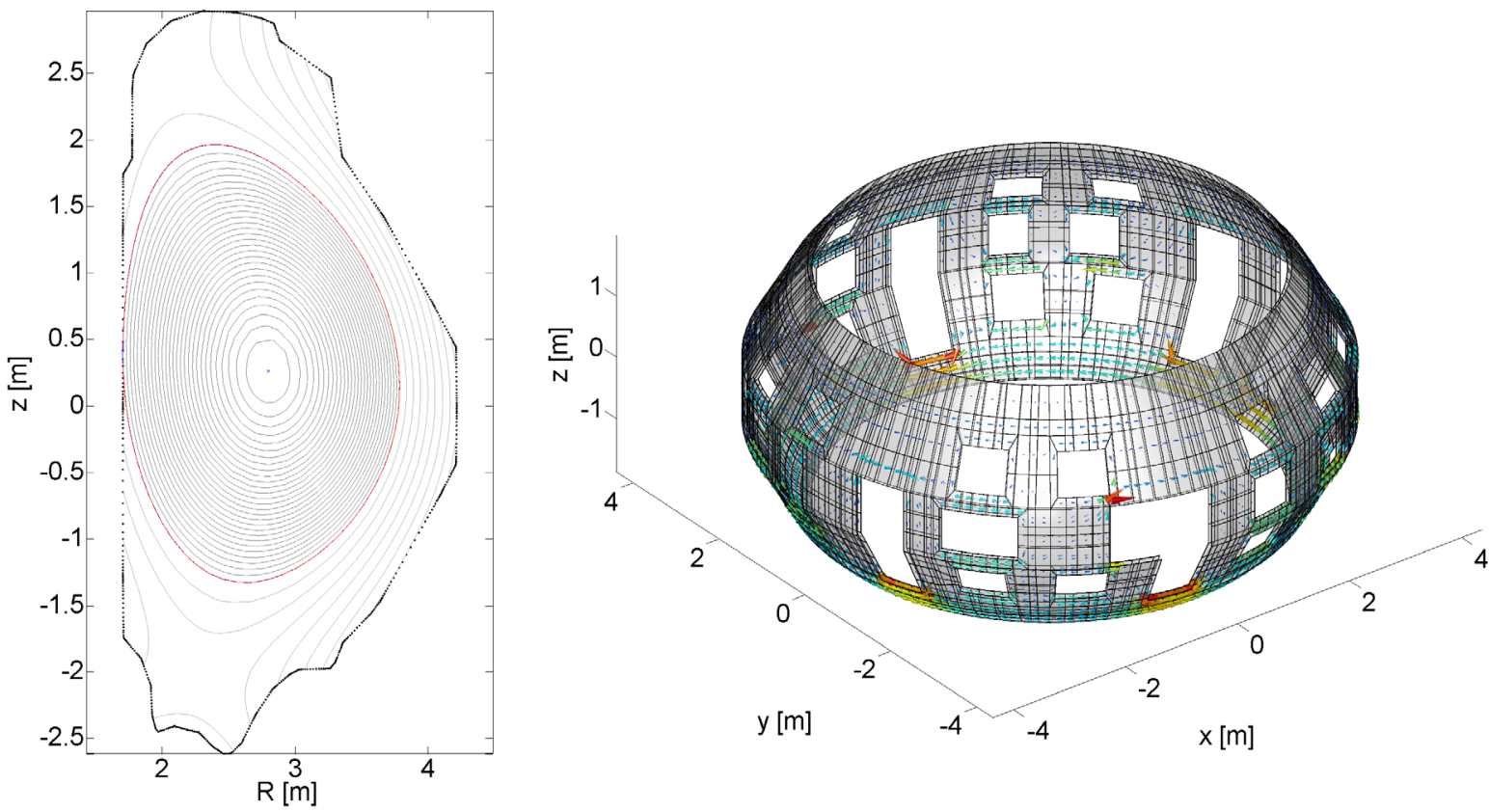

Figure 7. Example of uncontrolled plasma evolution following a beta drop, causing the plasma to hit the wall at the inboard side, as computed by the CarMa0NL code for parameters of Scenario 2. Left: plasma equilibrium. Right: patterns of eddy currents flowing in the stabilizing plate (normalized current densit, varying from 0 to 1 , is represented by a colour code, from blue to red). The finite elements mesh features also the vessel and the active control coils, which are not shown for clarity.

has a strong potential for machine protection and control, by line integrated electron density measurement via the CottonMouton effect [29]. Following the results of this feasibility study, implementation of a polarimetry system is being considered among the diagnostic upgrades for a further phase of the machine exploitation. A CAD drawing of a possible 12-chord arrangement of the polarimetry system is shown in figure 10 (left). Various options have been considered, with different number and locations of the viewing chords, including a minimal configuration with only three chords (one using a top port and two using an equatorial port). A DCN laser with wavelength of $195 \mu \mathrm{m}$ has been selected. The potential of the various configurations for q-profile reconstruction (and in particular the interest focused on the value of $q$ on axis, $q_{0}$ ) has been evaluated by means of the V3FIT [30] and VMEC [31] codes. To summarize the results, here we present the simulations obtained for the complete system ( 12 chords) and the minimum forseen configuration ( 3 chords). As shown in figure 10 , the minimum system has a high level of indetermination in terms of $q$-profile reconstruction, while the full system is much more effective. In both cases $q_{0}$ is the point with the largest indetermination, but the full system would already show the reversed magnetic shear in the core.

When evaluating these results, one should consider that they were obtained with a limited set of diagnostics information as the goal was to assess q-profile reconstruction capabilities comparing the different geometries of the polarimeter, taking as reference the requirement of $10 \%$ indetermination in the reconstruction of $q$. In the real case, for more refined studies, a full magnetic field reconstruction would use information from many more diagnostics (in the core other diagnosticsif available-will be very useful) which would most likely reduce the indetermination also in the core.

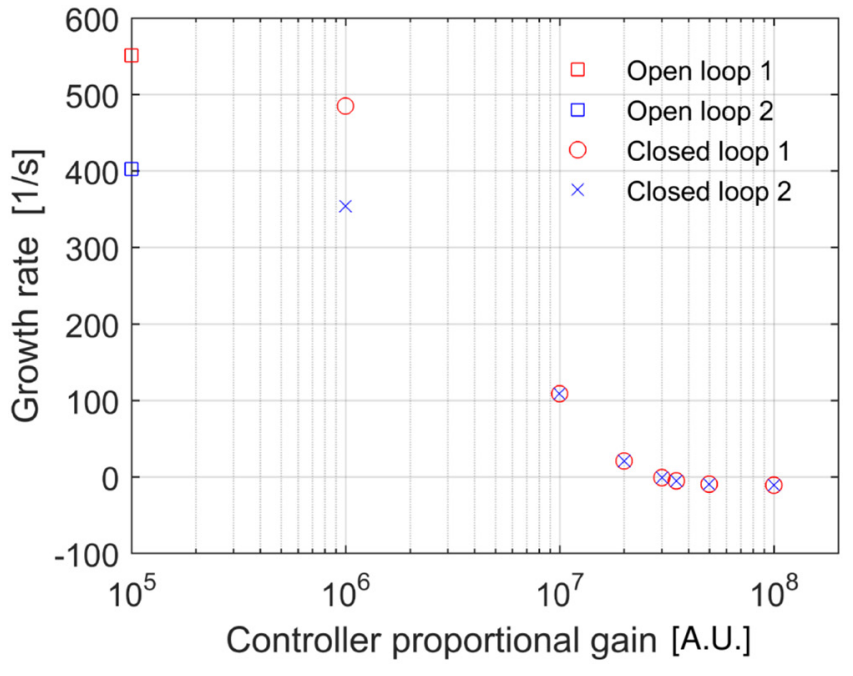

Figure 8. Example of RWM stabilization by a gain scan on the two unstable eigenmodes (labelled 1 and 2). The simulation uses six out of the eighteen available active coils, i.e. one toroidal array out of the three available (see e.g. figure 5 in [3] for more details on the JT-60SA Resistive Wall Mode Control Coil system).

3.1.2. Beam emission spectroscopy. The feasibility of a beam emission spectroscopy (BES) diagnostic system for measurement of the plasma turbulence in the JT-60SA machine configuration has been investigated [32]. The limiting factor of the BES diagnostic to be carefully evaluated is the spatial resolution of the diagnostic system with regard to the spatial and temporal scales of the detectable turbulent structures. This is influenced by three main components: the area of detector projection in the focal plane, the smearing caused by the atomic physics processes through the finite lifetime of the excited levels and the alignment of the lines of 

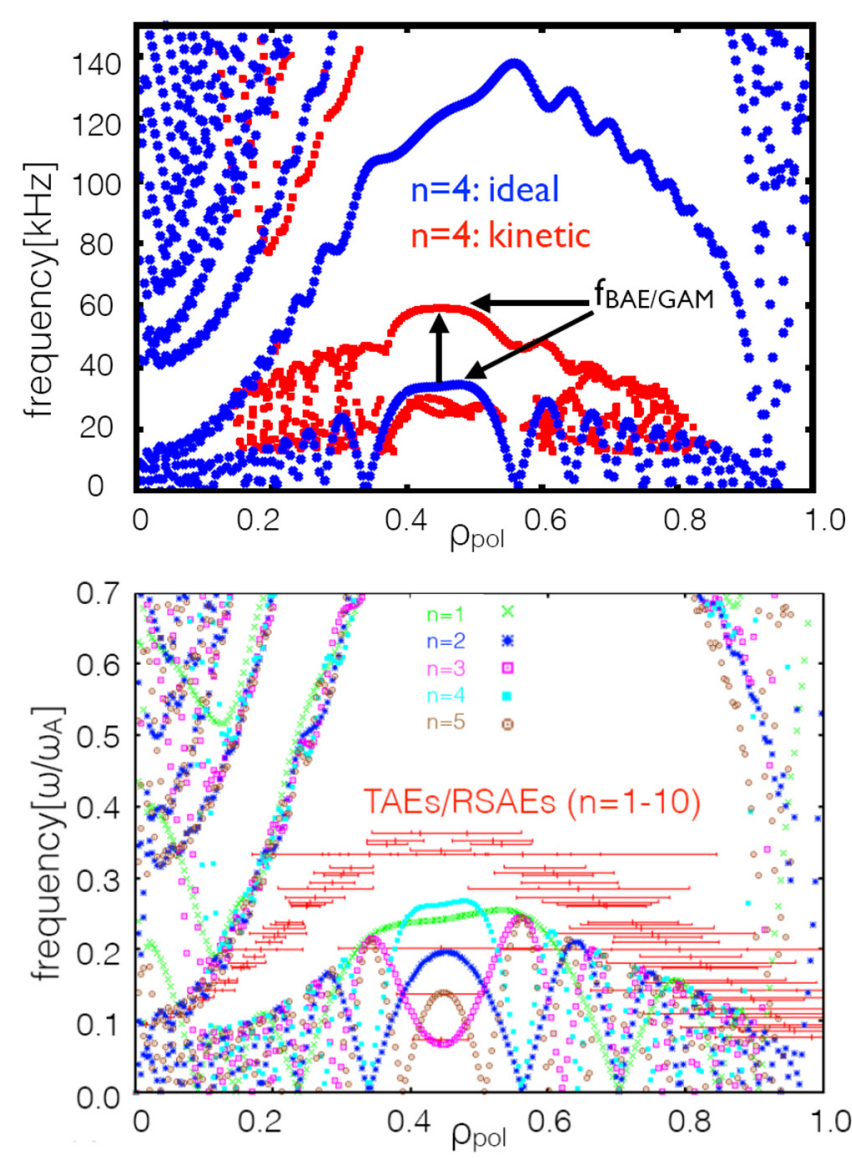

Figure 9. Alfvénic instability calculations by the linear gyrokinetic LIGKA code for Scenario 5-1. Top: ideal and kinetic continua for toroidal mode number $n=4$ (mode frequency versus radial coordinate $\rho_{\text {pol }}$, i.e. square root of normalised poloidal flux). Bottom: Shear Alfvén continua and gap modes: here, TAE and RSAE with toroidal mode numbers $n=1-10$ (mode frequency normalised to Alfvén frequency versus $\rho_{\text {pol }}$ ).

sight (LOS) to the magnetic field lines along which most fluctuating structures are elongated. Use of one of the deuterium heating neutral beams or of a dedicated beam injecting Alkali atoms has been considered. Both options have been modelled with the RENATE synthetic diagnostic, which is based on the collisional radiative model and calculates the expected light profile and spatial resolution [33]. Figure 11 shows modelling results for both sodium and lithium beams, i.e. the detected photon current profile (top panel) and peak photon count and emission smearing in function of beam energy (bottom panel). Both systems present a photon count that is sufficient and comparable within margins. Sodium beams have considerably lower penetration; however they have better spatial resolution due to lower emission smearing by atomic physics processes, allowing for a more detailed study of a narrow spatial domain. Both beam species show adequate emission in the scrape-off layer region, inferring a sodium set-up for SOL observation exclusively, while the lithium option would allow plasma edge observation as well.

Optimum choice for the observation geometry has been investigated for the tangential D beams used for plasma heating, for a possible BES system. Figure 12 shows possible locations of optimal observation points for tangential beam
8B located near the lower extreme of port P-17. The possible observation points within the boxed region correspond to a range of spatial resolutions of $2-3 \mathrm{~cm}$. This configuration is shown as an example of sufficient photon count for both edge and core observations, however the spatial resolution is not ideal and requires further optimization. Implementation of a BES system is now being considered among the diagnostic upgrades for a further phase of the machine exploitation.

3.1.3. Phase contrast imaging. Another turbulence diagnostic that is actively studied is phase contrast imaging (PCI). A system is being designed using a tangential viewing implementation and spatial filtering based on magnetic shearing in order to obtain localized information on both electron and ion scale turbulence [34]. Using a $10.6 \mu \mathrm{m} \mathrm{CO}_{2}$ laser and liquid nitrogen cooled detector, local turbulence measurements become possible. Figure 13(a) shows the geometry of the system: the beam is injected tangentially on the equatorial plane. As shown in figure $13(b)$, almost the entire radial region is covered by the beam, including low and high field side. The angle of the magnetic field projected to the perpendicular plane of the beam is shown in figure 13(c); calculations have been performed for a double-null divertor configuration, of the type of Scenario 1. The variation of the projected angle improves the spatial resolution: tangential viewing makes the variation larger and good resolution is achieved. As shown in figure $13(d)$, better resolution is expected for higher wavenumber. Measurements in almost the entire region of the turbulence become possible. This PCI system will contribute to the experimental investigation of the turbulence properties and to validation of numerical simulations of the turbulence driven transport.

3.1.4. Fast ion loss detector. In view of the already discussed importance of energetic particles distribution and confinement for JT-60SA scenarios, conceptual design studies of a fast ion loss detector (FILD) system [35] are being carried out, in strong synergy with analogous developments for ITER. In fact, it is remarkable that the relevant dimensionless parameters (i.e. fast particles beta and ratio of their velocity to Alfvén speed) of the JT-60SA negative NBI-driven fast ions are in the same range as those of alpha particles in ITER (see, for instance, figure 16 of [4]). Such a diagnostic would be able to resolve the lost fast ion distribution in both energy and pitch-angle, which will be essential to investigate phenomena such as N-NBI current drive, interaction of fast ions with MHD modes, as well as 3D field effects, e.g. impact of error field and RWM control coils on the fast ion confinement. A scintillator based FILD acts as a magnetic spectrometer, dispersing fast ions onto a scintillator, with the strike points depending on their gyroradius and pitch-angle. The detection is made by a CCD camera and a photomultiplier array; the system is mounted on a reciprocating arm. In the framework of the feasibility study for JT-60SA, the predicted output of the diagnostic has been computed by a synthetic diagnostic code. The system is being designed in such a way that excellent resolution is expected in gyroradius and pitch-angle for both negative and positive NBI beams, which have injected 

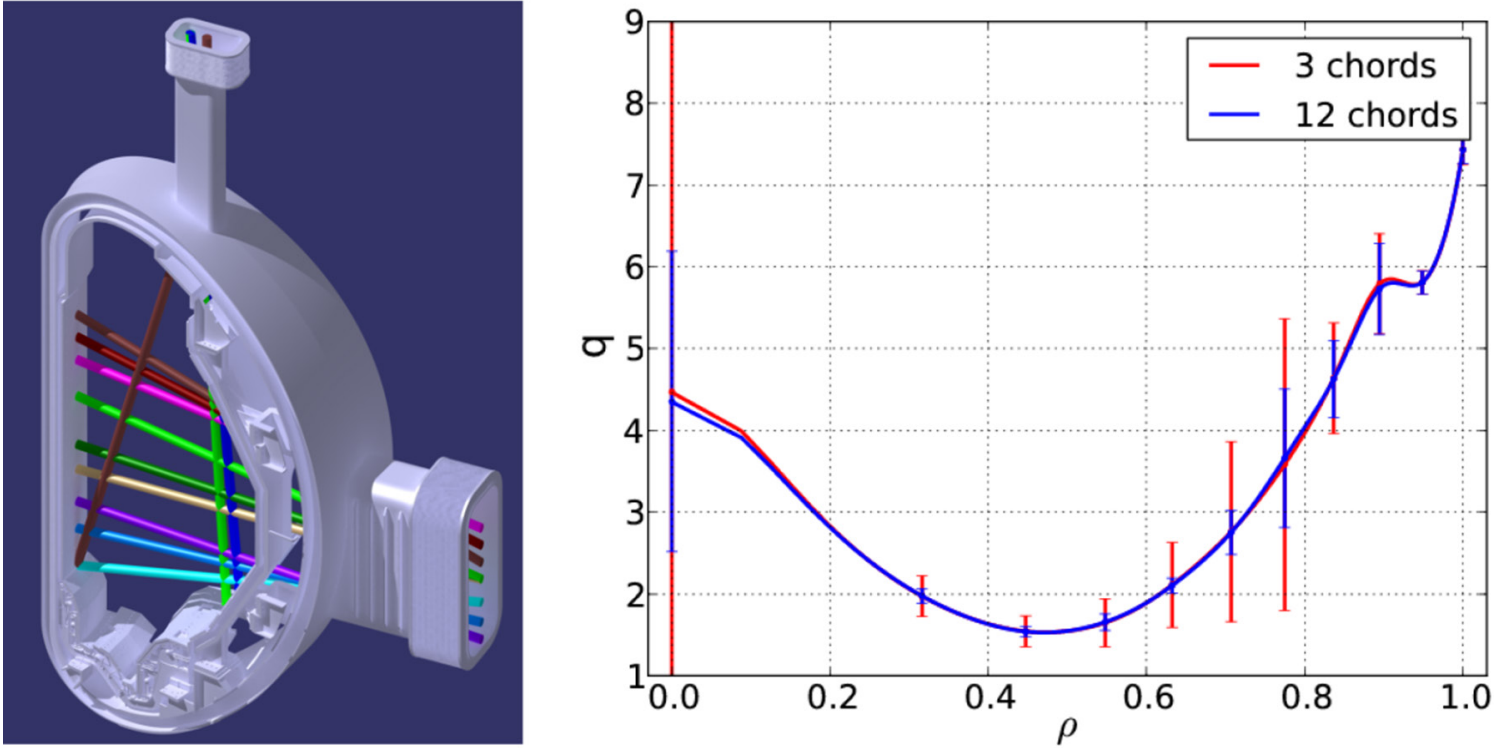

Figure 10. Left: drawing of a possible 12 viewing chords arrangement for the polarimetry system. Right: $q$-profile reconstruction (versus square root of normalised toroidal flux) by 3 or 12-chord polarimetry with error bars, for Scenario 5-1.
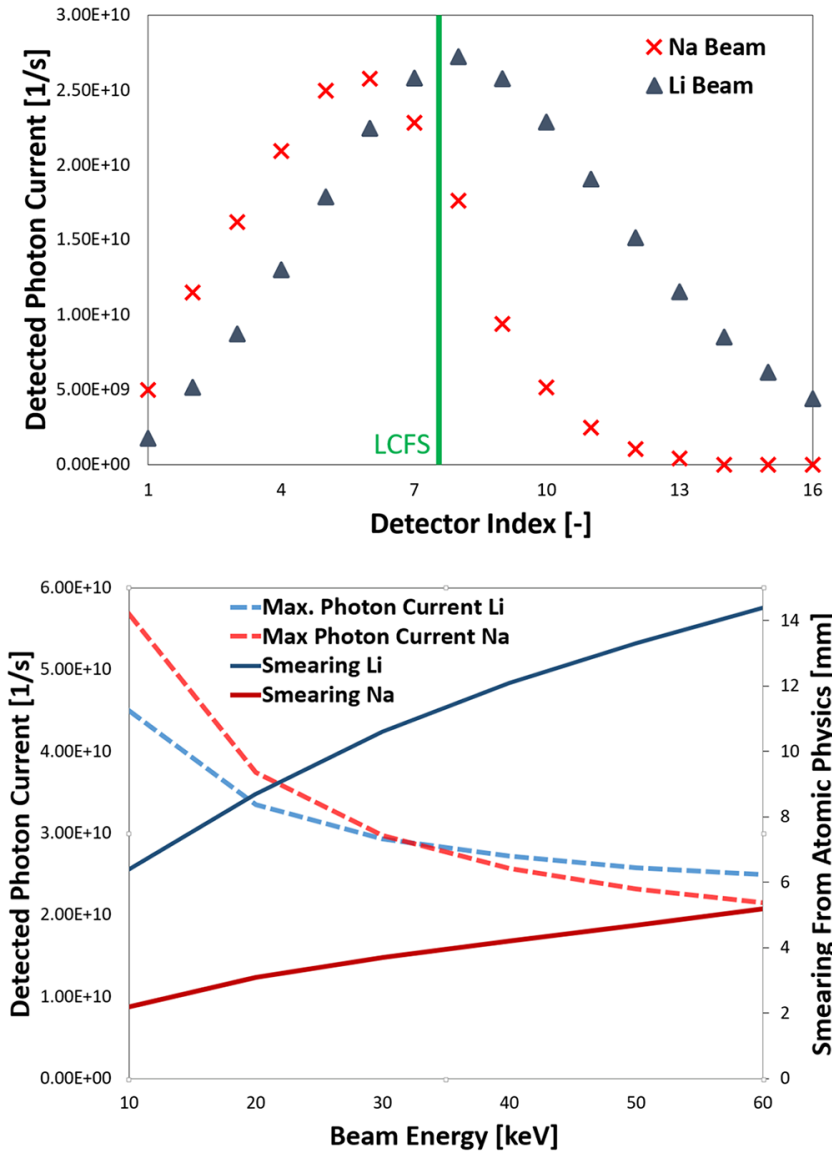

Figure 11. Evaluations for a possible BES system based on Alkali metal beam injection for parameters of Scenario 2. Comparison of Li (blue) and $\mathrm{Na}$ (red) beams in identical poloidal co-planar observation geometry. Top: Detected photon current profile, using a 16 pixel detector row with $1 \mathrm{~cm}$ pixel projection along the beam. Bottom: peak photon current and emission smearing as a function of beam energy.

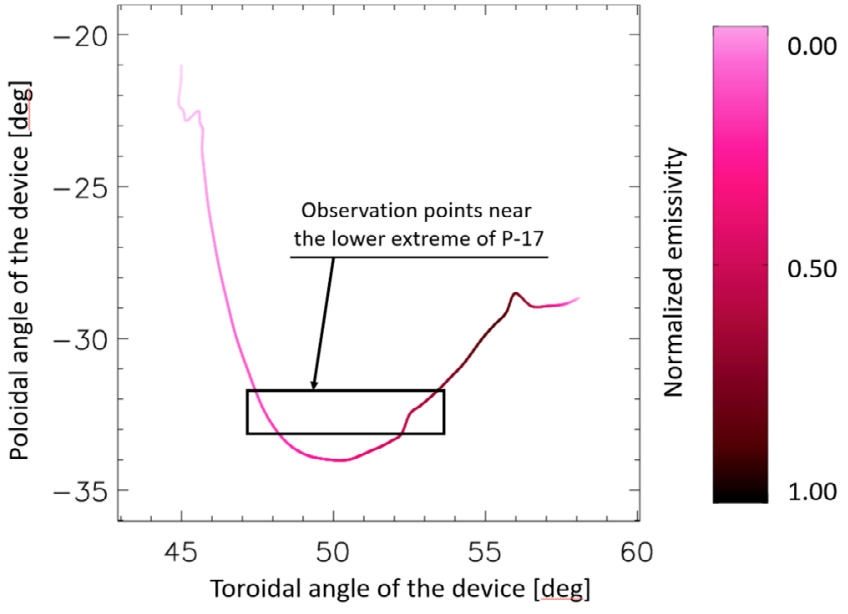

Figure 12. Evaluations for a possible BES system based on heating $\mathrm{D}$ beam injection for parameters of Scenario 2. Locations of observation points optimized for spatial resolution on torus surface for the tangential $85 \mathrm{keV}$ beam $8 \mathrm{~B}$.

energies of $500 \mathrm{keV}$ and $85 \mathrm{keV}$, respectively. An example of these synthetic diagnostic code results is shown in figure 14 . The FILD system being designed for JT-60SA is expected to be used and tested as a prototype of the future ITER system for alpha particle loss detection.

3.1.5. Fast wide-angle camera. Besides these long term developments of the JT-60SA diagnostic system, measurements that could be useful for the commissioning and first phase of operation are also being considered. Among them, a wide-angle version of the EDICAM visible camera [36] is being designed and will be procured as a direct European contribution to JT-60SA diagnostics. Several applications are possible: plasma breakdown observations (with detection of dangerous events, such as hot-spots and shinethrough), plasma 


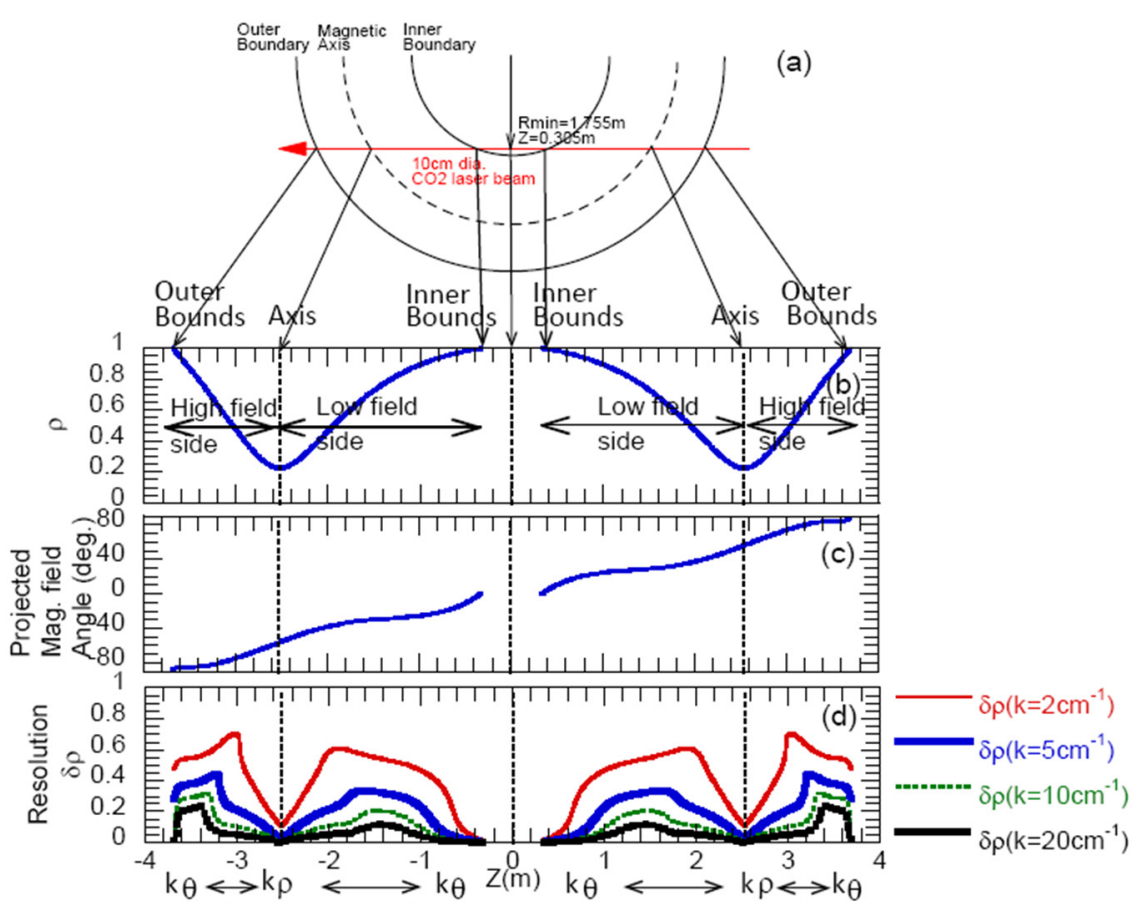

Figure 13. Spatial resolution of tangential PCI for JT-60SA double null divertor configuration. (a) Schematic view of the beam path, $(b)$ normalised radial position along the beam, $(c)$ projected magnetic field angle and $(d)$ spatial resolution. $Z$ coordinate is along the beam injection direction.
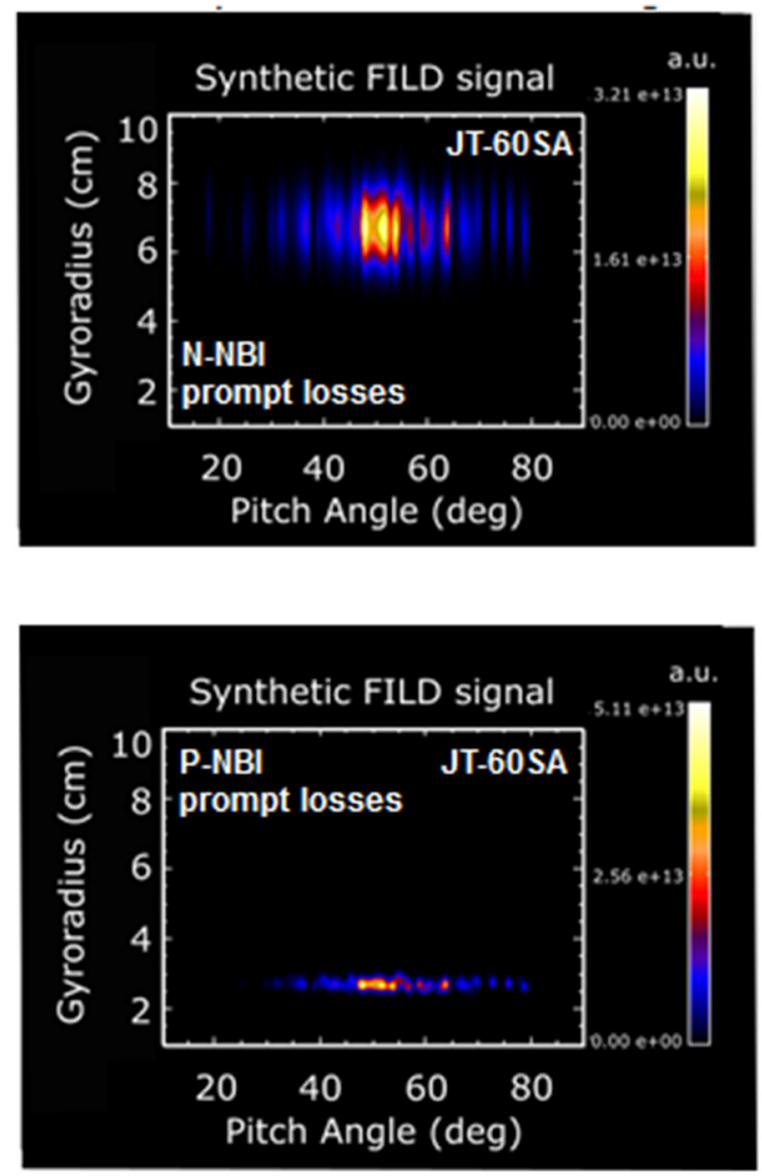

Figure 14. FILD signals simulated by the synthetic diagnostic code FILDSIM for fast ions driven by both negative (top) and positive (bottom) NBI.
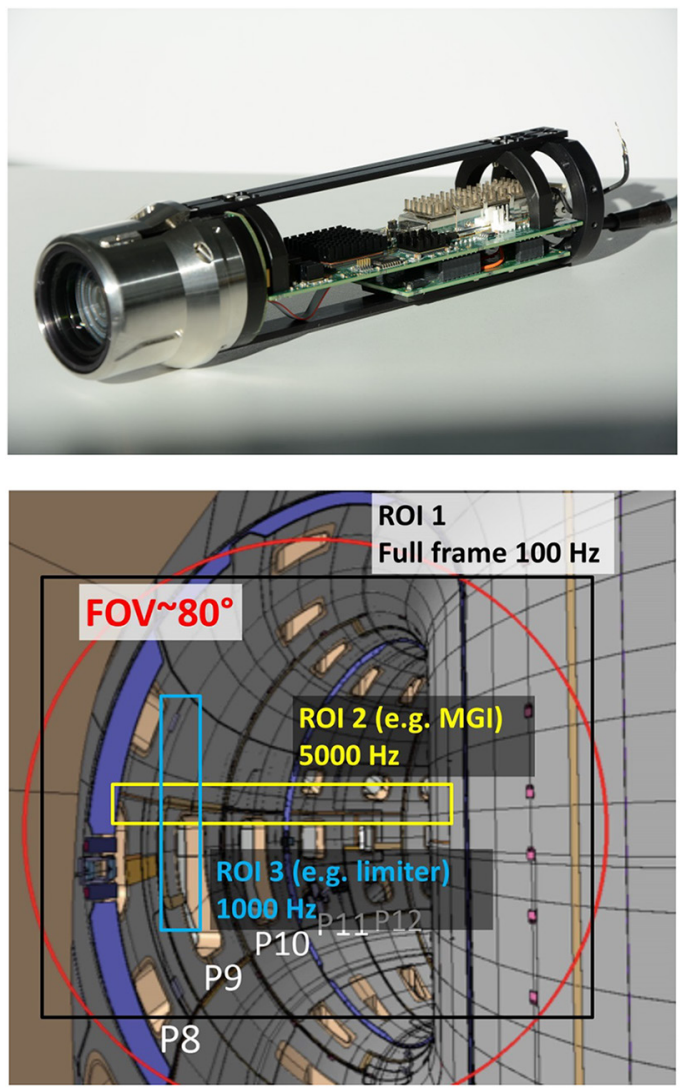

Figure 15. Top: EDICAM camera head, including pin-hole optics Bottom: example of possible EDICAM view with the camera region-of-interest (ROI) set-up for JT-60SA. This figure shows the EDICAM functionality of defining several ROIs in one camera. Field of view (FOV) is around $80^{\circ}$ 

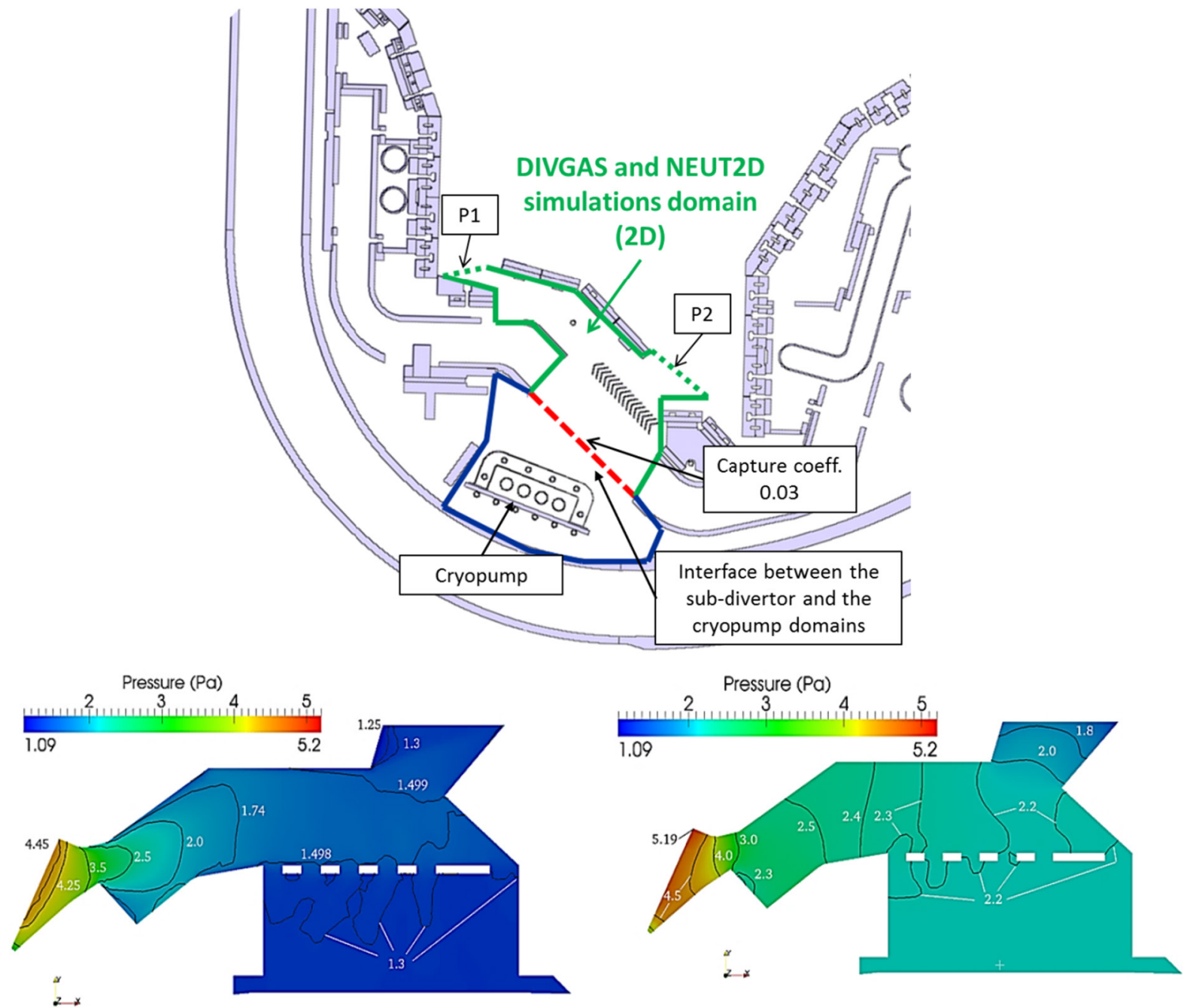

Figure 16. DIVGAS simulations of divertor pumping for Scenario 2. Top: divertor and sub-divertor structures and definition of the computational domains. The dashed red line indicates the chevron baffle which has been simulated by a structure with $50 \%$ transmission probability. Bottom: isobars in the sub-divertor region from a collisionless simulation (left) and including intermolecular collisions (right).

boundary identification with a temporal resolution up to $1 \mathrm{kHz}$ (comparable to magnetic equilibrium reconstruction), ELMs, disruptions and massive gas injection, SOL filaments statistical properties (correlation length, flow), in parallel to plasma overview measurements. In fact, a peculiar characteristic of this camera is that of providing measurements in multiple regions-of-interest (ROIs), which can be done simultaneously at different frame rates. This allows for the simultaneous capture of slow and fast image streams (up to 60000 frames s$^{-1}$ ), while keeping a low frame rate (e.g. 10-100 Hz) full resolution overview. In figure 15 a picture of the EDICAM camera head is shown (top panel) and an example of this multiple ROI capability is shown for the JT-60SA in vessel configuration (bottom panel). A version of this camera equipped with wideangle optics will be manufactured and installed on JT-60SA in order to be used from the first plasma.

\subsection{Matter injection and pumping}

3.2.1. Divertor pumping. One of the key elements of long pulse H-mode operation is the capability of mastering the particle balance by appropriate matter injection and pumping systems. In order to assess the operational window and to optimize the cryopump system design, extensive simulations of the divertor pumping system have been performed, with advanced numerical codes [37]. The impact of neutral gas dynamics on the particle removal process and the overall pumping efficiency in JT-60SA sub-divertor have been investigated by means of two different Monte-Carlo codes, with and without intermolecular collisions. As a first step, the EU code DIVGAS [38] and the Japanese code NEUT2D [39] have been successfully benchmarked without collisions. For this exercise, the computational domain has been derived from the CAD file, and simplified as illustrated in figure 16 (top). Boundary conditions (from SONIC calculations) were the pressures (fluxes, temperatures) at the two openings (P1, P2) and the interface to the cryopump, defined via a capture coefficient. Then, gas flow simulations have been performed with DIVGAS for a challenging case for pumping simulations, namely a high density scenario where collisional effects in the sub-divertor are most prominent [40]. An example of such simulations for Scenario 2 and the defined computational 

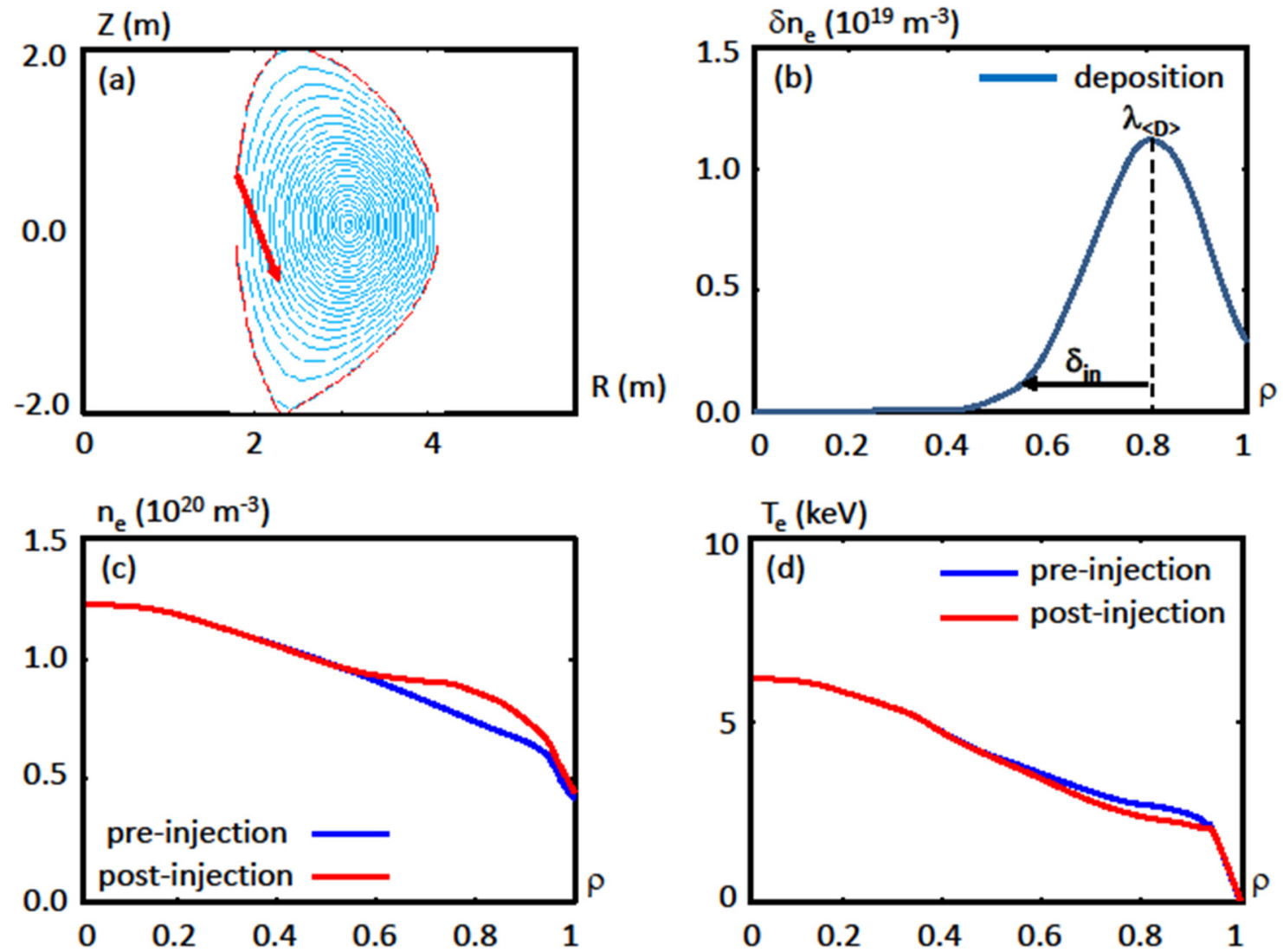

Figure 17. Pellet ablation simulation with the HPI 2 code, for Scenario 3, corresponding to the case ' $I n$ $470 \mathrm{~m} \mathrm{~s}^{-1}$, of table 4 . (a) Injection geometry; $(b)$ pellet deposition profile versus normalized radius; $(c)$ pre- and post-injection electron density profiles; $(d)$ the same for the electron temperature.

Table 4. Pellet ablation computations with the HPI 2 code, for various reference scenarios, injection location and pellet speeds. Pellet mass is $6.5 \times 10^{20} \mathrm{D}$ atoms; for Scenario 3, also the case of an oversized pellet $\left(4 \times 10^{21} \mathrm{D}\right.$ atoms $)$ is considered. Deposition profile parameters in normalized radius: $\lambda_{\langle\mathrm{D}\rangle}$ is the location of the maximum; $\delta_{\text {in }}$ is the $1 / 10$ width of the profile towards the plasma core (see figure $17(b)$ ).

\begin{tabular}{|c|c|c|c|c|c|c|}
\hline$\lambda_{\langle\mathrm{D} \gamma} / \delta_{\text {in }}$ & Scenario 1 & Scenario 2 & Scenario 3 & Scenario 4 & Scenario 5 & Scenario 6 \\
\hline Big $2000 \mathrm{~m} \mathrm{~s}^{-1}$ & - & - & $\mathbf{0 . 9 5} / 0.20$ & - & - & - \\
\hline Big $4000 \mathrm{~m} \mathrm{~s}^{-1}$ & - & - & $\mathbf{0 . 9 2} / 0.32$ & - & - & - \\
\hline Out $2000 \mathrm{~m} \mathrm{~s}^{-1}$ & $\mathbf{0 . 9 6 / 0 . 1 3}$ & $\mathbf{0 . 9 6 / 0 . 1 2}$ & $\mathbf{0 . 9 5 / 0 . 1 5}$ & $\mathbf{0 . 9 7 / 0 . 1 7}$ & $\mathbf{0 . 9 3} / 0.33$ & $\mathbf{0 . 9 2 / 0 . 2 2}$ \\
\hline Out $4000 \mathrm{~m} \mathrm{~s}^{-1}$ & $\mathbf{0 . 9 5} / 0.20$ & $\mathbf{0 . 9 5} / 0.21$ & $\mathbf{0 . 9 3} / 0.51$ & $0.96 / 0.38$ & $\mathbf{0 . 9 3} / 0.50$ & $\mathbf{0 . 7 2} / 0.34$ \\
\hline Top $470 \mathrm{~m} \mathrm{~s}^{-1}$ & $\mathbf{0 . 9 8 / 0 . 1 1}$ & $\mathbf{0 . 9 8 / 0 . 0 8}$ & $\mathbf{0 . 9 8} / 0.09$ & $0.97 / 0.10$ & $\mathbf{0 . 9 8} / 0.13$ & $\mathbf{0 . 9 8} / 0.15$ \\
\hline Top $2000 \mathrm{~m} \mathrm{~s}^{-1}$ & $\mathbf{0 . 9 8} / 0.10$ & $\mathbf{0 . 9 8} / 0.08$ & $\mathbf{0 . 9 8} / 0.08$ & $\mathbf{0 . 9 8} / 0.08$ & $\mathbf{0 . 9 8} / 0.08$ & $\mathbf{0 . 9 8} / 0.12$ \\
\hline In $200 \mathrm{~m} \mathrm{~s}^{-1}$ & $\mathbf{0 . 8 2} / 0.32$ & $\mathbf{0 . 8 6} / 0.38$ & $\mathbf{0 . 8 4} / 0.24$ & $\mathbf{0 . 8 6} / 0.26$ & $\mathbf{0 . 7 7} / 0.24$ & $\mathbf{0 . 7 3} / 0.27$ \\
\hline In $470 \mathrm{~m} \mathrm{~s}^{-1}$ & $\mathbf{0 . 7 7 / 0 . 3 4}$ & $\mathbf{0 . 8 2} / 0.37$ & $\mathbf{0 . 8 1} / 0.31$ & $\mathbf{0 . 8 3} / 0.31$ & $\mathbf{0 . 7 2} / 0.22$ & $\mathbf{0 . 6 9} / 0.26$ \\
\hline
\end{tabular}

domain is presented in figure 16 (bottom), showing isobar contours computed without and with intermolecular collisions, which display significantly enhanced pressure in the sub-divertor region when collisions are taken into account. Finally, the cryopumps have been characterized in terms of heat loads and operational requirements derived from the results of the sub-divertor calculations.

3.2.2. Pellet injection. In the framework of the pellet system conceptual design studies [41], ablation and fuelling simulations have been carried out by means of the HPI2 code [42], in order to develop high-density pellet-fuelled scenarios and to assess the system capabilities for different injection geometries (inboard, outboard, top) and pellet speeds (200-4000 $\mathrm{m} \mathrm{s}^{-1}$ ). An example of the pellet deposition profile and its effect on density and temperature profiles for the high-density Scenario 3 is shown in figure 17. Results on pellet penetration for all the scenarios and pellet characteristics considered are summarized in table 4. It appears that the injection configuration is quite important for the pellet fuelling characteristics and inboard launch provides the best suitable solution despite its speed restriction. Note that in order to fully assess the fuelling capability of the system, the effect of ELMs should also be taken into account. A feasibility study of a Massive Gas Injection system has also started. Such a system would be of great importance for qualifying the methods and parameters 
to be used in ITER, where disruption mitigation will be indispensable.

\subsection{ECRH system}

In the JT-60SA tokamak four Electron Cyclotron wave launchers will be installed and used for local heating, current drive and plasma initiation by injection of high-power and long-pulse waves into the plasma by double-frequency gyrotrons at 110 and $138 \mathrm{GHz}$ [43]. The same gyrotrons can also be used at $82 \mathrm{GHz}$ for short pulses $(<1 \mathrm{~s})$, especially for EC wall cleaning. In order to characterize the optical and physical performances of the ECRH system in the full steering range, the antenna has been modelled and the beams simulated with the numerical electromagnetic code GRASP [44] that offers the possibility to calculate the electromagnetic scattering from a general structure, including sequences of plane and curved reflectors [45]. An example of such calculations is given in figure 18 (top), where a sketch of the ECRH antenna layout and location is shown, together with colour contour plots of the far field antenna pattern in the plasma. Using the actual antenna mirror geometry, injection angle range and the launched beam pattern, the beam-tracing code GRAY [46] has then been used for a full functional analysis of the ECRH system, in particular for its use as a localised current drive tool, e.g. for NTM control. For the case of Scenario 2 and wave frequency $138 \mathrm{GHz}$, the ECCD functional diagram is shown in figure 18 (bottom), displaying contours of the driven current (for $1 \mathrm{MW}$ injected power) and of the location of the driven current maximum versus poloidal and toroidal injection angles. These types of diagrams are used as a basis for the design of scenarios in which ECCD plays a significant role, either for current density profile tailoring or for tearing mode control.

The use of the ECRH system, in particular for plasma initiation, requires a careful analysis of the EC stray radiation, both with modelling in the various conditions of low absorption and with studies of the possible detection systems. Such studies have been carried out [47], estimating the average power loading as the whole transmitted power distributed over the projection of the astigmatic beam on the chamber wall at the reflecting points. The poloidal angle range corresponding to the antenna mechanical limits is considered, i.e. $-40^{\circ}<\alpha<+20^{\circ}$, where $0^{\circ}$ is the horizontal direction from the launching mirror location shown in figure 18, positive angles correspond to aiming towards the top and negative towards the bottom. Two reflections on the vessel wall or on the stabilizing plate are considered as the main source of direct damage. The peak and the average wave power densities (in MW m${ }^{-2}$ ) versus the poloidal angle for both 110 and $138 \mathrm{GHz}$ with pure perpendicular injection are plotted in figure 19 at the first and second reflection. As a general feature, the power density at the second bounce is about $1 / 3$ the power density at first bounce. Moreover, the highest load at the first bounce is found as expected for normal incidence. In the case of second bounce, the worst cases are found at $-20^{\circ}$ (shortest path and thus lower beam diffraction) and at $-40^{\circ}$ (corresponding to
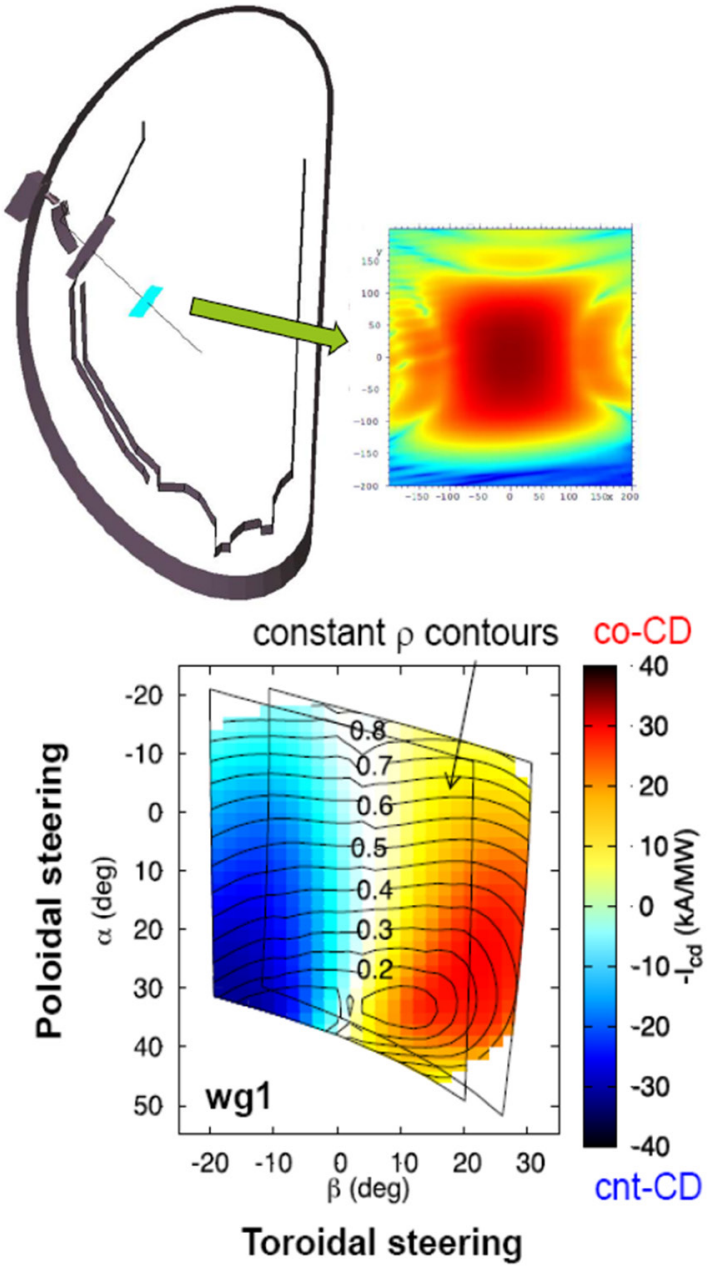

Figure 18. Modelling of EC beams and driven current for Scenario 2 and $138 \mathrm{GHz}$. Top: example of far field antenna pattern computed with the GRASP code (first side lobes are at $-25 \mathrm{~dB}$ ). Bottom: EC driven current and absorption location computed with the beamtracing code GRAY for the full poloidal and toroidal steering range.

normal incidence on the stabilising plate). These results are being used to design a proper detection and protection system.

\subsection{Transition to tungsten PFCs}

In the present strategy of the JT-60SA exploitation, an initial phase of operation will be carried out with carbon wall and divertor, a machine configuration expected to be more favourable for attaining the nominal scenarios, including the most challenging ones at high beta and full non-inductive current. In a later phase, all the PFCs will be replaced with metallic ones, in order to provide closer support to ITER and to be more relevant for DEMO. In order to prepare this phase, a feasibility study on the transition to $\mathrm{W}$ divertor and first wall is ongoing. In addition to the previously mentioned edge and scenario simulations with $\mathrm{C}$ environment $[11,12,14,15]$, simulations have started with $\mathrm{W}$ environment. An example of results obtained by the COREDIV code is shown in figure 20 for Scenario 2 and three different seeding gases. It is found that with $\mathrm{W}$ divertor the power delivered to the divertor plates 


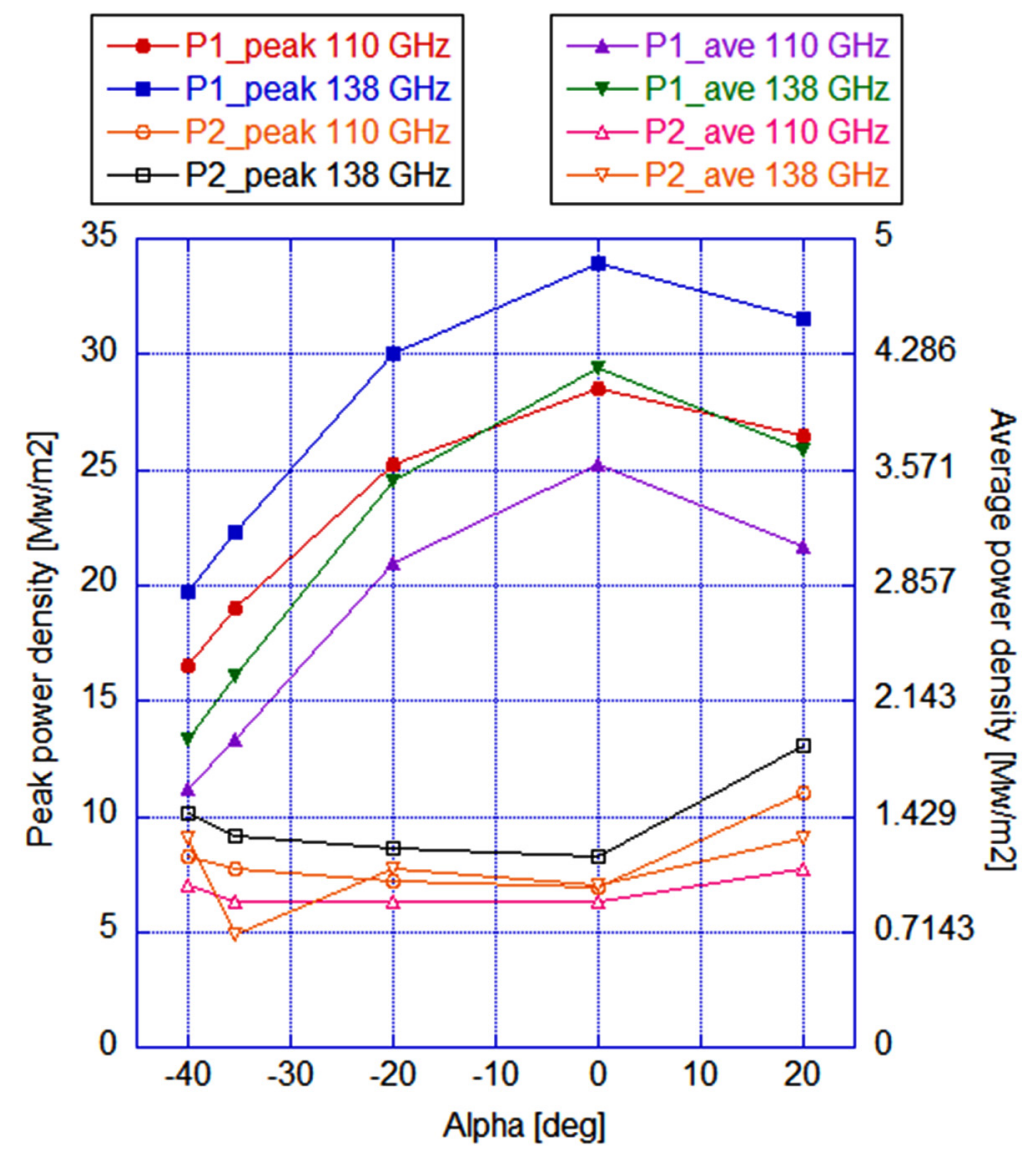

Figure 19. Computed peak and average EC power densities at the wall versus poloidal injection angle (Alpha) for zero toroidal angle and with no plasma absorption. P1: first reflection; P2: second reflection.

without seeding is very high and using a seeding gas to control energy exhaust is mandatory. For high density (Scenario 3) a wide operation window with Ne seeding is found $[12,48]$. For Scenario 2, which is at lower density, the operation window is narrower, however significant radiation fractions (as shown by figure 20) and tolerable heat loads to the target plates $(<10$ MW $\mathrm{m}^{-2}$ ) can still be obtained for acceptable values of $Z_{\text {eff }}$ $(<3)$. Argon seeding appears to be the optimal choice.

In addition to these simulations, technical feasibility is also addressed. In particular, one of the main issues for transition to W PFC in JT-60SA is the choice between massive W components versus $\mathrm{W}$ coated ones. Thick $\mathrm{W}$ plating on graphite or $\mathrm{CFC}$ is now being considered and looks promising. Successful high heat flux tests of $0.5 \mathrm{~mm}$ vacuum plasma sprayed coatings produced in Japan on the ion beam facility GLADIS, located at IPP/Garching, have been carried out in the framework of these joint activities. Such tests included hundreds of 3-4 s cycles at power flux of $15 \mathrm{MW} \mathrm{m}^{-2}$, leading to temperatures up to $2000^{\circ}$, with no observation of melting, cracking or delamination.

\section{Operation}

\subsection{EC wall cleaning}

Wall conditioning will be required in JT-60SA to control fuel and impurity recycling and to improve plasma performance

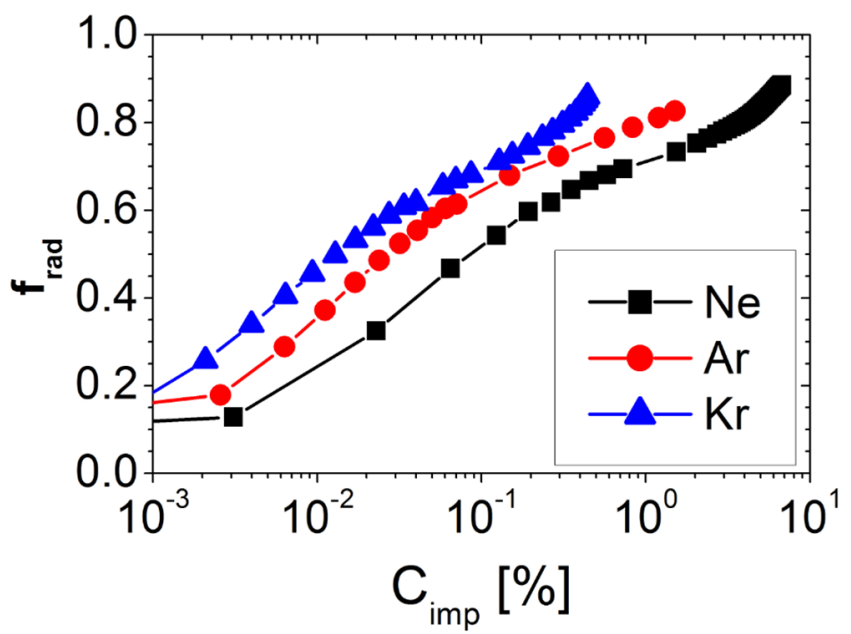

Figure 20. COREDIV simulations with $\mathrm{W}$ PFC for Scenario 2 and various seeding gases. Radiation fraction versus $\mathrm{Ne}, \mathrm{Ar}$ and $\mathrm{Kr}$ concentrations.

and reproducibility. Because of the superconducting magnetic field, glow discharge cleaning will not be usable between shots (as in ITER) and electron cyclotron wall conditioning (ECWC) is envisaged, a technique that is not fully validated yet, in particular at the 2nd EC harmonic. To this end, dedicated experiments [49] have been performed on the TCV tokamak, in the framework of the joint EU-Japan activities and of the EUROfusion experimental programme on Medium Size 
$B_{v}=0,60 \% x B_{T}$
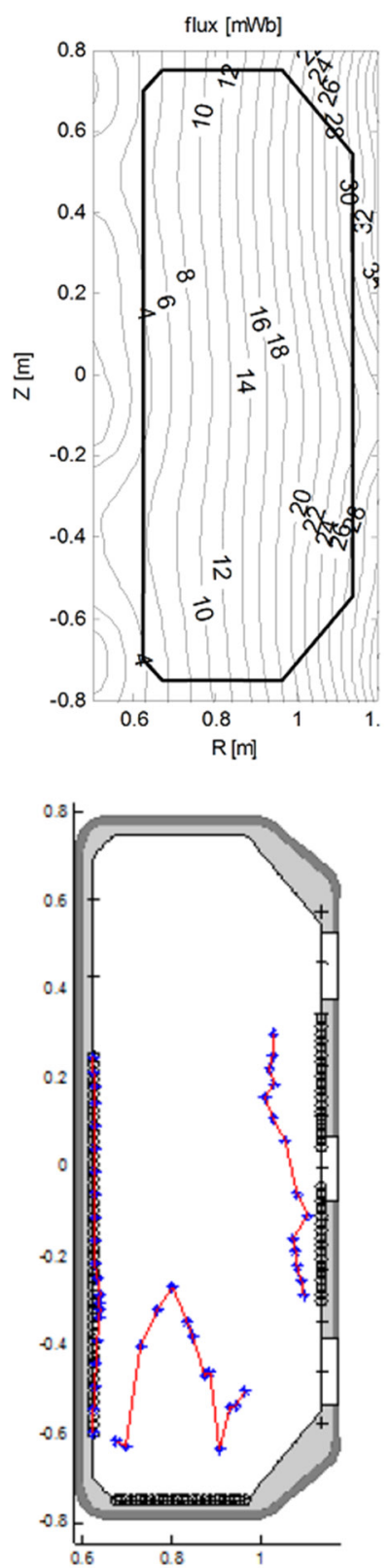

$B_{V}+2 B_{R}$
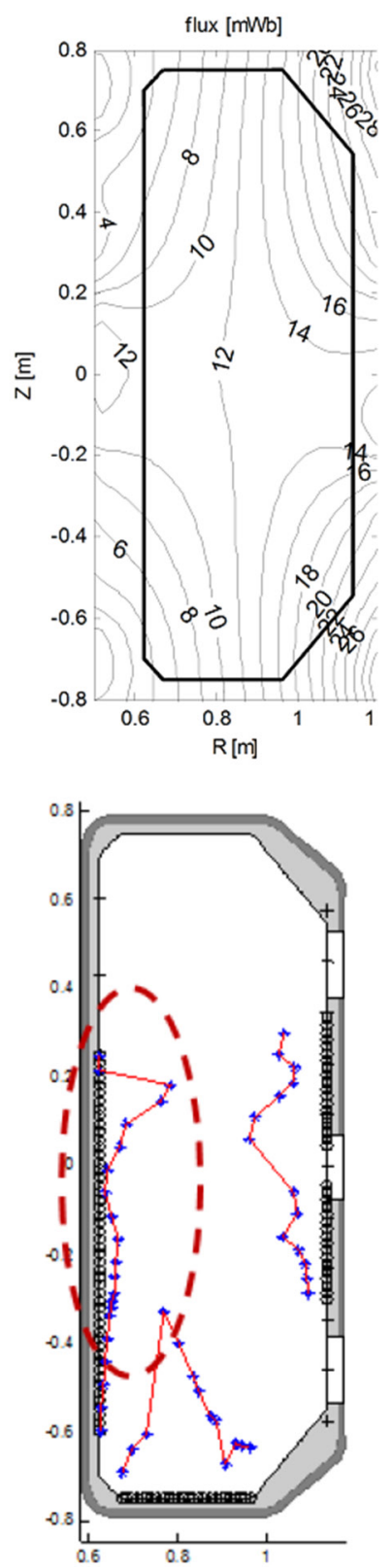

$\frac{3}{2} B_{v}+3 B_{R}$
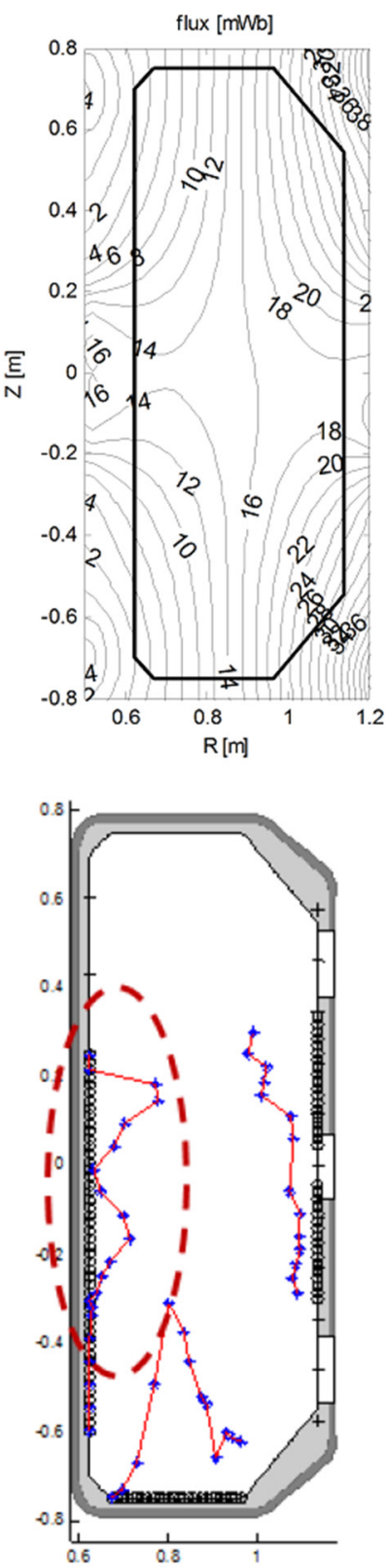

Figure 21. ECWC He discharges on TCV, at $B_{\mathrm{T}}=1.3 \mathrm{~T}, p=10^{-2} \mathrm{~Pa}, P_{\mathrm{EC}}=480 \mathrm{~kW}$. Top: poloidal field patterns as reconstructed from the values of the currents in the sixteen shaping coils. Bottom: ion saturation currents from the wall Langmuir probes for different combinations of $B_{\mathrm{H}}$ and $B_{\mathrm{V}}$.

Tokamaks. Nearly sixty Helium conditioning discharges have been successfully produced, at a toroidal field $B_{\mathrm{T}}=1.3$ or 1.54 $\mathrm{T}$, with gyrotrons at $82.7 \mathrm{GHz}$ in the extraordinary mode, mimicking ECWC operation in JT-60SA at the second harmonic of the EC wave. ECRH was injected for $2 \mathrm{~s}$, with powers between 90 and $480 \mathrm{~kW}$. Discharge parameters were tuned in order to (i) minimize the time for the onset of ECWC plasmas, thus minimizing absorption of stray radiation by in-vessel components; (ii) improve discharge homogeneity by extending the discharge vertically and radially, as well as wall coverage, in particular of inboard surfaces where JT-60SA plasmas will be initiated; (iii) assess the efficiency of He-ECWC to deplete carbon walls from fuel. An optimized combination of vertical and radial magnetic fields, with amplitudes typically $0.1-0.6 \%$ of that of $B_{\mathrm{T}}$, has been determined, which resulted in lower breakdown time, improved wall coverage and enhanced fuel removal. This is illustrated in figure 21, showing poloidal field patterns (top) and ion saturation currents from the wall 


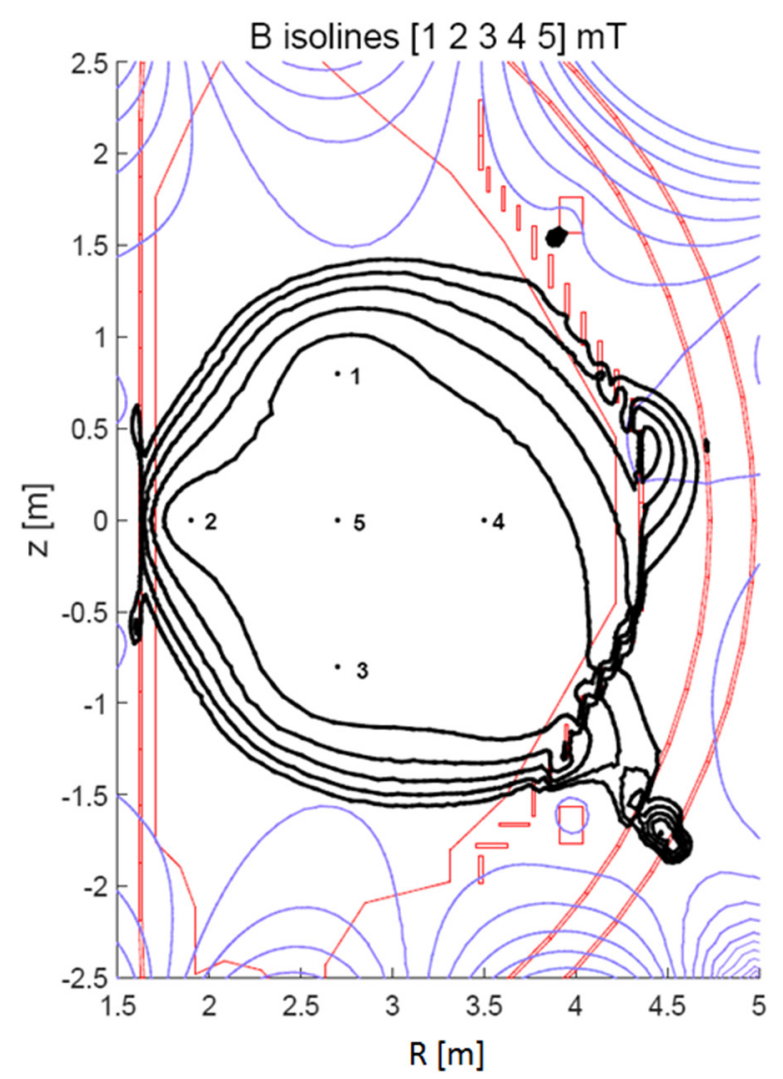

Figure 22. Poloidal magnetic field flux (blue) and modulus of poloidal field (black) maps at the breakdown time, computed by CREATE-L. Dots with numbers indicate the control points, where the poloidal field is kept to a minimum. The red curves are the modelled machine structures (vessel, stabilizing plate, coils).

Langmuir probes (bottom) for different combinations of radial and vertical magnetic fields. As a result of this optimization, standard Ohmic $\mathrm{D}_{2}$-plasma could be then sustained, whereas it would not have been possible without He-ECWC.

\subsection{Breakdown studies}

Optimum breakdown conditions have been explored by various magnetic simulation tools. An example of magnetic flux and field maps computed by means of the CREATE-L code [50] is shown in figure 22. Dots with numbers describe the desired breakdown region, where the controllers minimize the modulus of the poloidal field. The implementation of the machine structure (coils, vessel, stabilising plate) in EU codes allows comparing with analogous simulations developed with Japanese codes $[51,52]$ and in general to search for optimum control strategies, as discussed in section 4.3.

ECRH assisted breakdown modelling studies have been carried out using the code BKD0 [53], solving a set of balance equations for energy and particles together with the circuit equation for the plasma current, to estimate the temporal evolution of plasma parameters. Wave trajectories are computed with the beam tracing code GRAY [46], including reflection by the wall facing the antenna. This analysis has allowed quantifying the amount by which the operational pressure domain is extended when using EC assisted breakdown, as well as the impact of reflections and of the ECRH pulse duration. The results are illustrated by figure 23 , showing that the maximum pre-fill pressure for successful breakdown increases by approximately $0.5 \mathrm{mPa}$ per $\mathrm{MW}$ of additional injected power, when a poloidal injection angle is used allowing reflection of the EC beam through the breakdown region.

\subsection{Magnetic simulation and control tools}

Various tools for magnetic equilibrium computation are being applied to JT-60SA discharge simulation, with specific controller developments, aiming at efficient control of plasma current, position, shape and vertical stability. A magnetohydrodynamic equilibrium control simulator (MECS) has been developed to study techniques for plasma equilibrium control in JT-60SA. The MECS code combines an isoflux controller with the Cauchy condition surface (CCS) method for reconstruction of the plasma shape, including calculation of the eddy currents flowing in the tokamak structures [54]. The CCS method uses the positions of the control points received from the controller and evaluates the poloidal flux at the last closed flux surface (LCFS) and at a set of defined control points. Plasma equilibrium control during the heating phase has been simulated in order to test the capability of this control scheme to maintain a constant plasma shape while normalised poloidal pressure and internal inductance $\left(\beta_{\mathrm{p}}\right.$ and $l_{\mathrm{i}}$ ) evolve, as shown in figure 24 . In the simulation without the CCS method, the quantities required for plasma equilibrium control are calculated directly from the equilibrium. It is found that accurate control of the plasma can be achieved by this method by using an appropriate choice of the control gains in order to mitigate the influence of the LCFS identification error.

Other control architectures for vertical stabilisation, plasma current and shape control have been designed and tested by a set of tools based on the CREATE-L and CREATE-NL equilibrium codes, including models of the poloidal field coils power supplies [55]. In particular, two possible solutions have been specified for the vertical stabilisation: a simple twogain controller (which may be an effective solution if adaptive algorithms will be deployed), and a time-optimal one. As far as the plasma current and shape control is concerned, an isoflux multi-input-multi-output controller has been proposed, based on the JET extreme shape controller approach [56]. For diverted plasmas, such an approach minimizes the mean square error between the flux at the control points and the flux at the $X$-point. As an example, figure 25 shows the results obtained with the proposed control architecture, for the simulation of the plasma current ramp-up (from 1.7 MA to 5.5 MA) of Scenario 2. During the ramp-up, it is assumed that an L-H transition occurs, leading to significant increase 


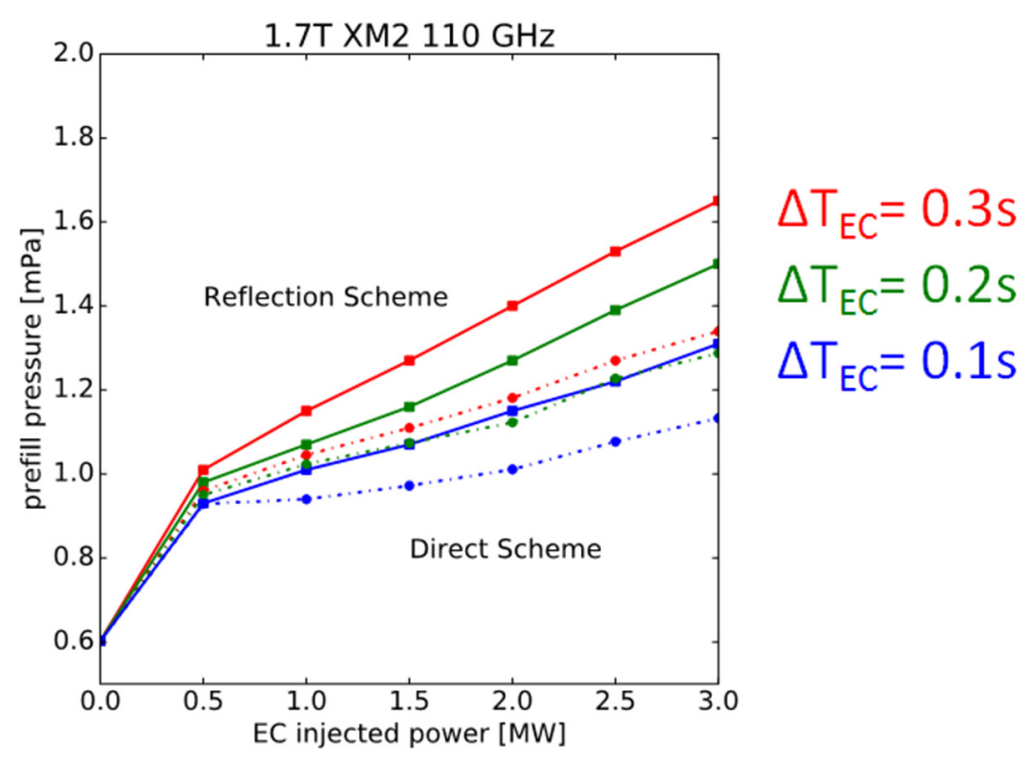

Figure 23. EC-assisted breakdown computations with the BKD0 code. Maximum pre-fill pressure for successful start-up versus injected EC power, with (solid lines) and without (dashed lines) a wall reflection. $\Delta T_{\mathrm{EC}}$ is the $\mathrm{EC}$ pulse duration.
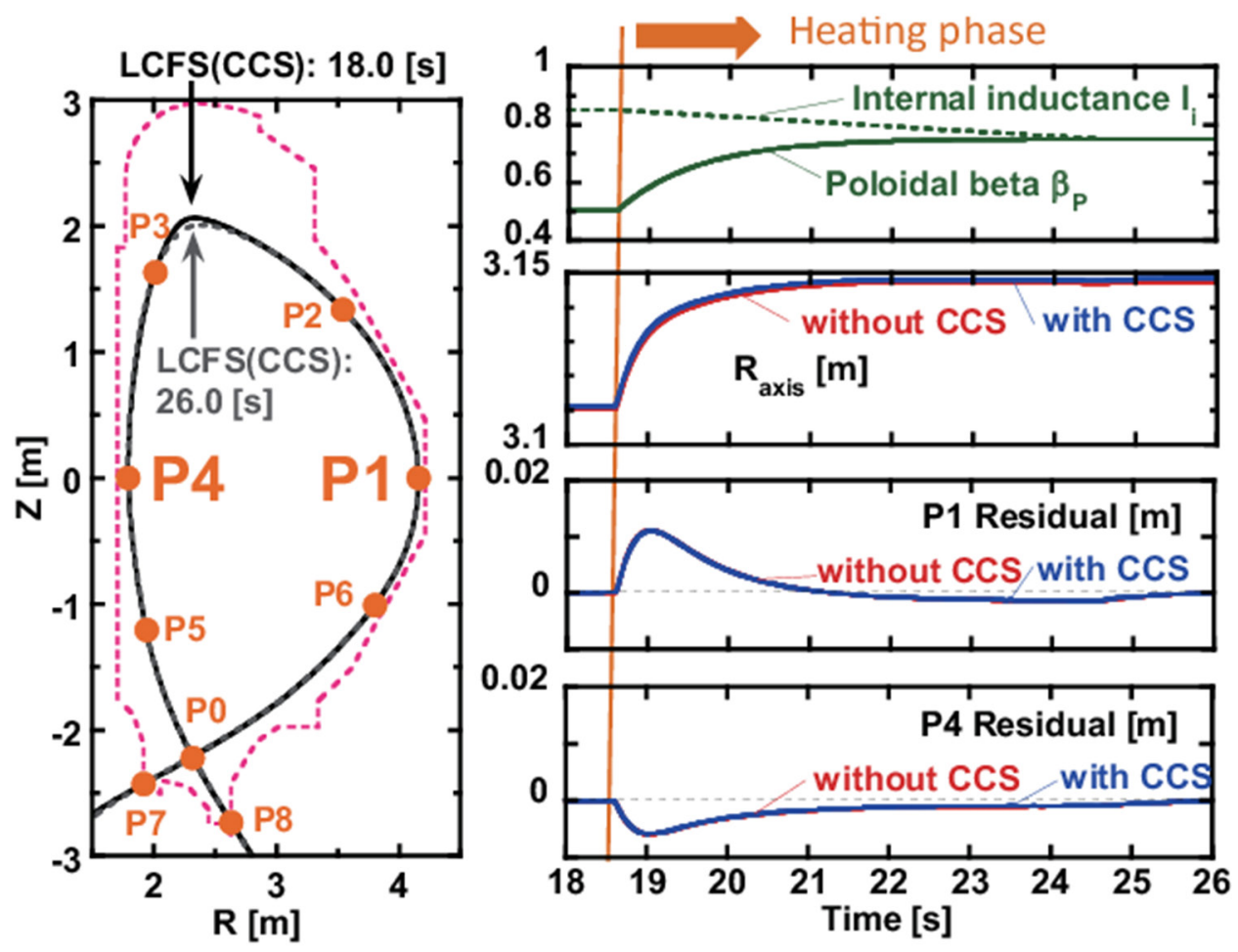

Figure 24. Plasma equilibrium control simulated by the MECS code for an $I_{\mathrm{p}}=5.5 \mathrm{MA}$ scenario. Left: locations of the control points and LCFS by CCS at two times. Right: waveforms of (from top to bottom) $\beta_{\mathrm{P}}$ and $l_{\mathrm{l}}, R_{\text {axis }}, \mathrm{P} 1$ and P4 residuals without and with CCS. In the simulation without the CCS method, the quantities required for plasma equilibrium control are calculated directly from the equilibrium.

of $\beta_{\mathrm{p}}$, which acts as a disturbance for the plasma magnetic control. In particular, the time traces of the fluxes at the control points and at the $X$-point are shown in the top right panel, while two snapshots of the plasma poloidal cross section are shown in the bottom part. Note that, for the considered setup, the isoflux loop controls the $R$ and $Z$ position of the $X$-point, together with the flux error at the 10 defined control points. Comparison of these sets of tools and control schemes, independently developed in Japan and EU is now ongoing, the first steps being reported in [55].

\subsection{Data and analysis tools}

The JT-60SA data and analysis system is being developed, following modern principles and methods, optimized for operation by an international team, i.e. including remote 

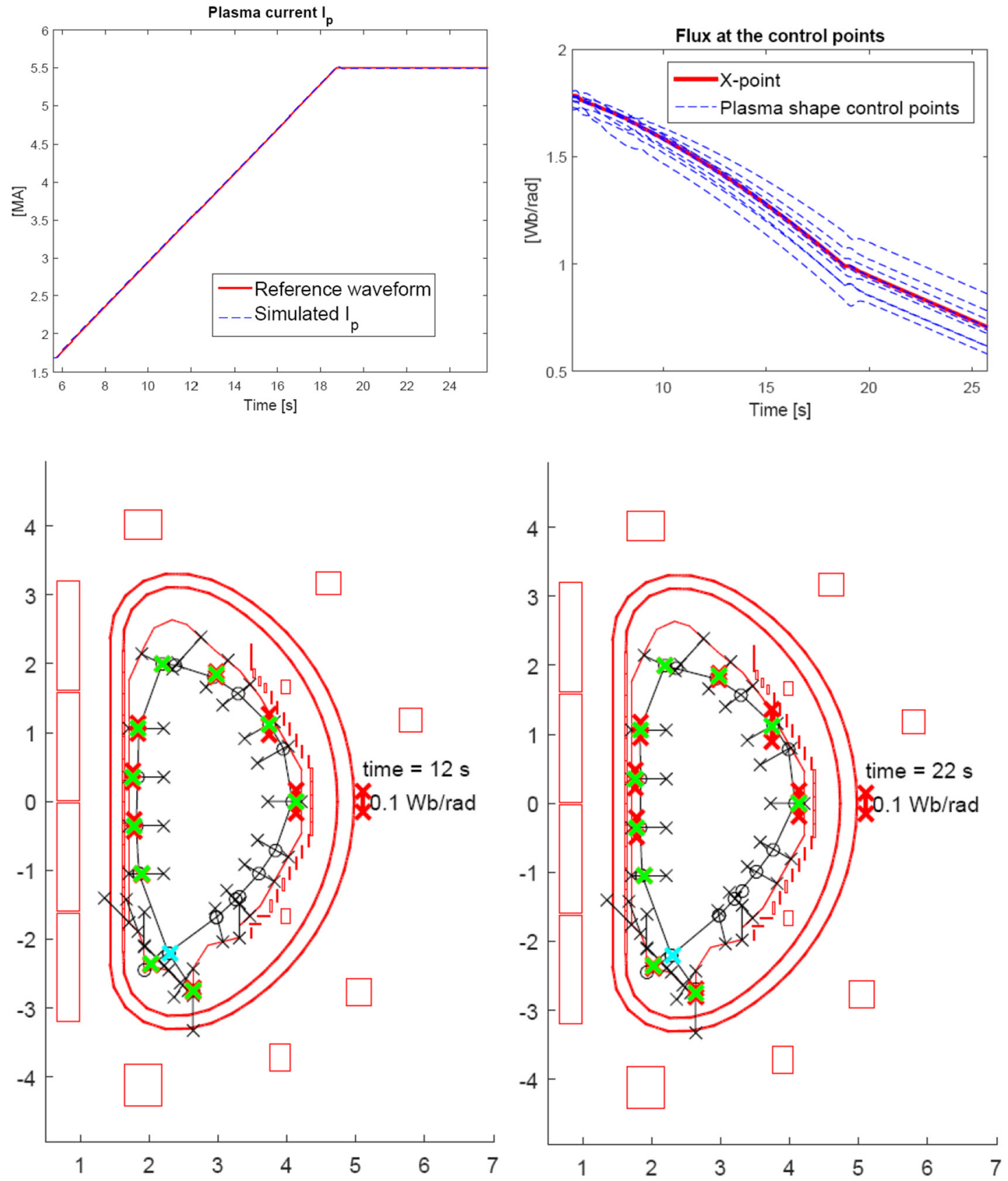

Figure 25. Closed loop simulation of the plasma current ramp-up (from 1.7 MA to 5.5 MA) of Scenario 2, performed using the CREATE-L linear model. Isoflux control achieves plasma shape control by keeping at zero the difference between the flux at the control points and the flux at the $X$-point. Top left: plasma current versus time in the ramp-up phase. Top right: magnetic flux at the $X$-point and at the plasma shape control points. Bottom: plasma poloidal cross section during the plasma current ramp-up $(t=12 \mathrm{~s}$, left) and at the flat-top $(t=22 \mathrm{~s}$, right). The blue cross shows the desired position for the $X$-point, while the green crosses show the isoflux control points (i.e. the points where the flux error is controlled to zero); these control points are selected along some of the available black control segments. The red segments delimited by crosses are used to show the control error in $\mathrm{Wb} \mathrm{rad}^{-1}$. Note that the maximum error is about $0.1 \mathrm{~Wb}^{\mathrm{rad}}{ }^{-1}$.

participation tools. The main requirements of such a system have been collected and critically discussed [57]: data model, data archiving and architecture, remote computers and data access, support tools for pulse preparation and execution, data analysis, results discussion, publication preparation, etc. Implementation of the IMAS [58] system (i.e. the ITER data and analysis suite) is considered, which would make of JT-60SA a full scale test bed of the future ITER scientific exploitation system. Demonstration of remote participation using experiments on EU tokamaks should take place in the near future, before the start of JT-60SA experiments, under the framework of the ITER Remote Experimentation Centre [59].

\section{Conclusions and prospects}

As the start of integrated commissioning and operation (scheduled for 2020) approaches, the coordinated Japan-EU activities for the preparation of JT-60SA exploitation enter now a phase characterized by important milestones. The main 
one is the elaboration of a 'final' version of the Research Plan (v4.0), namely the reference document to be used for defining the programme of the first experimental campaigns. This version is intended to take fully into account the most recent evolutions of the ITER Research Plan, because now the revised ITER schedule gives ample opportunities for substantial contributions by JT-60SA. Other milestones will be strongly connected to operation oriented activities: more and more detailed modelling of scenarios, including transients and controls, completion of design of sub-systems for the first phase of operation (in particular selected diagnostics, matter injection systems, cryopumps), precise definition of data and control systems, remote participation tools, elaboration of the structure and organisation of the experimental campaigns.

\section{Acknowledgments}

This work has been carried out within the framework of the EUROfusion Consortium and has received funding from the EURATOM research and training programme 2014-2018 under grant agreement No 633053. The views and opinions expressed herein do not necessarily reflect those of the European Commission. The authors gratefully acknowledge members of the JT-60SA Integrated Project Team for data exchange and fruitful discussions.

\section{ORCID}

G. De Tommasi (ㄷ) https://orcid.org/0000-0002-8509-7176

K. Galazka (1) https://orcid.org/0000-0001-8445-2044

L. Garzotti (1) https://orcid.org/0000-0002-3796-9814

L. Pigatto (1) https://orcid.org/0000-0002-0556-0440

\section{References}

[1] Shirai H. et al 2017 Nucl. Fusion 57102002

[2] JT-60SA Research Unit, JT-60SA Research Plan 2016 www.jt60sa.org/pdfs/JT-60SA_Res_Plan.pdf, v3.3

[3] Shirai H. et al 2016 Fusion Eng. Des. 109-11 1701

[4] Kamada Y. et al 2011 Nucl. Fusion 51073011

[5] Garcia J. et al 2014 Nucl. Fusion 54093010

[6] Honda M. and Fukuyama A. 2006 Nucl. Fusion 46580

[7] Hayashi N. et al 2010 Phys. Plasmas 17056112

[8] Artaud J.F. et al 2010 Nucl. Fusion 50043001

[9] Wakatsuki T. et al 2015 Plasma Phys. Control. Fusion 5765005

[10] Aiba N. et al 2006 Comput. Phys. Commun. 175269

[11] Zagórski R. et al 2016 Nucl. Fusion 56016018

[12] Zagórski R. et al 2017 Nucl. Fusion 57066035

[13] Kawashima H. et al 2016 Contrib. Plasma Phys. 56778

[14] Hayashi N. et al 2016 Core-edge coupled predictive modeling of JT-60SA high-beta steady-state plasma with impurity accumulation Nucl. Fusion submitted

[15] Romanelli M. 2017 Investigation of sustainable high- $\beta$ scenarios in JT-60SA C-wall Nucl. Fusion (https://doi. org/10.1088/1741-4326/aa7ad4)
[16] Suzuki Y. et al 2014 3D plasma response to resonant external magnetic perturbation and its impact on fast ion confinement in JT-60SA plasmas 25th IAEA Fusion Energy Conf. (St. Petersburg, Russia, 13-18 October 2014) p P7-37 (www-naweb.iaea.org/napc/physics/FEC/FEC2014/ fec2014-preprints/332_THP737.pdf)

[17] Snyder P. et al 2009 Phys. Plasmas 16056118

[18] Shiraishi J. et al 2016 Impact of kinetic effects of energetic particles on RWM stability in rotating high-beta plasmas 26th IAEA Fusion Energy Conf. Nucl. Fusion submitted

[19] Liu Y. et al 2008 Phys. Plasmas 15112503

[20] Pigatto L. et al 2016 Resistive wall mode stability in JT-60SA high $\beta_{\mathrm{N}}$ scenarios 43rd EPS Conf. on Controlled Fusion and Plasma Physics (Leuven, Belgium, 4-8 July 2016) p P4.078 (http://ocs.ciemat.es/EPS2016PAP/pdf/P4.078.pdf)

[21] Mastrostefano S. and Villone F. 2016 Nonlinear 3D analysis of JT-60SA $\mathrm{n}=0$ instabilities 43rd EPS Conf. on Controlled Fusion and Plasma Physics (Leuven, Belgium, 4-8 July 2016) p P4.079 (http://ocs.ciemat.es/EPS2016PAP/pdf/ P4.079.pdf)

[22] Mastrostefano S. et al 2015 Fusion Eng. Des. 96-7 659

[23] Villone F. et al 2012 Plasma Phys. Control. Fusion 54085003

[24] Villone F. et al 2013 Plasma Phys. Control. Fusion 55095008

[25] Bettini P. et al 2015 IEEE Trans. Magn. 517204105

[26] Bolzonella T. et al 2016 Securing high $\beta_{\mathrm{N}}$ JT-60SA operational space by MHD stability and active control modelling Preprint: 2016 IAEA Fusion Energy Conf. (Kyoto, Japan, 17-22 October 2016) p P1-18 (https:// nucleus.iaea.org/sites/fusionportal/Shared\%20Documents/ FEC\%202016/fec2016-preprints/preprint0468.pdf)

[27] Lauber P. et al 2007 J. Comput. Phys. 226447

[28] Todo Y. et al 2005 Phys. Plasmas 12012503

[29] Boboc A. et al 2015 Rev. Sci. Instrum. 86091301

[30] Hanson J.D. et al 2009 Nucl. Fusion 49075031

[31] Hirshman S.P. and Whitson J.C. 1983 Phys. Fluids 263554

[32] Asztalos Ö. et al 2016 Feasibility study on the JT-60SA tokamak beam emission spectroscopy diagnostics 29th Symp. on Fusion Technology (Prague, Czech Republic, 5-9 September 2016) p P1.074

[33] Guszejnov D. et al 2012 Rev. Sci. Instrum. 83113501

[34] Tanaka K. et al 2016 Rev. Sci. Instrum. $8711 \mathrm{E} 118$

[35] Garcia-Muñoz M. et al 2016 Rev. Sci. Instrum. 87 11D829

[36] Zoletnik S. et al 2013 Fus. Eng. Des. 881405

[37] Day C. et al 2016 Assessment of the operational window for JT-60SA divertor pumping under consideration of the effects from neutral-neutral collisions Preprint: 2016 IAEA Fusion Energy Conf. (Kyoto, Japan, 17-22 October 2016) p P4-42 (https://nucleus.iaea.org/sites/fusionportal/ Shared\%20Documents/FEC\%202016/fec2016-preprints/ preprint0312.pdf)

[38] Day C. et al 2014 Fusion Eng. Des. 891505

[39] Shimizu K. et al 2003 J. Nucl. Mater. 313-6 1277

[40] Gleason-Gonzalez C. et al 2016 Fusion Eng. Des. 109-11 693

[41] Lang P. et al 2016 Conceptual design of the JT-60SA pellet launching system 29th Symp. on Fusion Technology (Prague, Czech Republic, 5-9 September 2016) p P2.019

[42] Pégourié B. et al 2007 Nucl. Fusion 4744

[43] Kobayashi T. et al 2015 Fus. Eng. Des. 96-7 503

[44] Pontoppidan K. (ed) 2005 GRASP9 Technical Description, TICRA Engineering Consultants, Copenhagen

[45] Platania P. et al 2015 Optical and physical performances evaluations for the JT-60SA ECRF antenna AIP Conf. Proc. 1689090010

[46] Farina D. 2007 Fusion Sci. Technol. 52154

[47] Moro A. et al 2016 Electron cyclotron stray radiation detection and machine protection system proposal for 
JT-60SA Fusion Eng. Des. (https://doi.org/10.1016/j. fusengdes.2017.03.028)

[48] Galazka K. et al 2017 Plasma Phys. Control. Fusion 59045011

[49] Douai D. 2016 Development of helium electron cyclotron wall conditioning on TCV for the operation of JT-60SA Nucl. Fusion submitted

[50] Albanese R. and Villone F. 1998 Nucl. Fusion 38723

[51] Urano H. et al 2015 Fusion Eng. Des. 100345

[52] Shinya K. 2000 J. Plasma Fusion Res. 76479
[53] Granucci G. et al 2015 Nucl. Fusion 55093025

[54] Miyata Y. et al 2014 Plasma Fusion Res. 93403045

[55] Cruz N. et al 2017 Control Eng. Pract. 6381

[56] Ariola M. and Pironti A. 2005 IEEE Control Syst. Mag. 2565

[57] Innocente P. et al 2015 Fus. Eng. Des. 96-7 799

[58] Imbeaux F. et al 2015 Nucl. Fusion 55123006

[59] Ozeki T. et al 2016 Fus. Eng. Des. 1121055 